\title{
Functionalized extracellular matrix scaffolds loaded with endothelial progenitor cells promote neovascularization and diabetic wound healing
}

Siqi $\mathrm{He}^{1,2}$, Tanaya Walimbe ${ }^{3}$, Hongyuan $\mathrm{Chen}^{2}$, Kewa Gao ${ }^{1,2,5}$, Priyadarsini $\mathrm{Kumar}^{2,5}$, Yifan Wei ${ }^{2}$, Dake $\mathrm{Hao}^{2,5}$, Ruiwu Liu ${ }^{4}$, Diana L Farmer ${ }^{2,5}$, Kit S.Lam ${ }^{4}$, Jianda Zhou ${ }^{1}$, Alyssa Panitch ${ }^{2,3}$, Aijun Wang ${ }^{2,3,5 *}$

${ }^{1}$ Department of Burns and Plastic Surgery, The Third Xiangya Hospital of Central South University, ${ }^{2}$ Department of Surgery, UC Davis, ${ }^{3}$ Department of Biomedical Engineering, UC Davis, ${ }^{4}$ Department of Biochemistry and Molecular Medicine, UC Davis, ${ }^{5}$ Institute for Pediatric Regenerative Medicine, Shriners Hospitals for Children

\begin{abstract}
Diabetic ischemic wound treatment remains a critical clinical challenge. Strategies that enhance angiogenesis and improve ischemic pathology may promote the healing of poor wounds, particularly diabetic wounds in highly ischemic condition. We previously identified a cyclic peptide LXW7 that specifically binds to integrin $\alpha v \beta 3$ on endothelial progenitor cells (EPCs) and endothelial cells (ECs), activates VEGF receptors, and promotes EC growth and maturation. In this study, we designed and synthesized a pro-angiogenic molecule LXW7-DS-SILY by conjugating LXW7 to a collagen-binding proteoglycan mimetic DS-SILY and further employed this novel bifunctional ligand to functionalize extracellular matrix (ECM) scaffolds, promote neovascularization and accelerate ischemic wound healing. We established a Zucker Diabetic Fatty (ZDF) rat ischemic skin flap model and found the wounds treated by LXW7-DS-SILYfunctionalized ECM scaffolds, with or without EPCs, significantly improved wound healing, enhanced neovascularization and modulated collagen fibrillogenesis. These functionalized ECM scaffolds also significantly promoted EPC attachment, growth and survival in the ischemic environment. Altogether, this study provides a promising novel treatment to accelerate diabetic ischemic wound healing, thereby reducing limb amputation and mortality of diabetic patients.
\end{abstract}

Keywords: diabetic ischemic wound, ECM scaffold, endothelial progenitor cells neovascularization, wound healing 


\section{Introduction}

In 2019, the International Diabetes Federation (IDF) estimated a global population of 415 million people with diabetes mellitus, and that the number of people affected will increase to 700 million by 2045 [1]. IDF also estimated that $25 \%$ of this population will develop diabetic foot ulcers (DFU) in their lifetime [2], with over 65\% of DFUs having an ischemic pathology prone to serious infections, leading to diabetes-related lower extremity amputations [3]. Moreover, ulcers and other foot complications contribute greatly to diabetes-related hospitalizations, and thus represent a significant healthcare burden. Accelerating the wound healing process in ischemic diabetic foot ulcers would reduce the need for lower extremity amputation, improve survival of patients suffering from DFUs, and decrease diabetes-related hospitalizations.

The current standard of care for DFU treatment includes debridement of the wound, antibiotic management of any infection, ulcer off-loading, and revascularization surgeries, if indicated. The current bioengineered scaffold therapies using commercially available scaffolds such as Dermagraft (human fibroblast-derived dermal substitute) and Regranex (recombinant PDGF, also known as becaplermin gel), or tissue engineered skin, but all have disadvantages due to multiple repeat applications for efficacy, secondary surgeries, delayed healing, fibrosis [4]. And none of them target re-vascularization specifically. To overcome these difficulties, stem cellbased therapy to improve revascularization of the tissue has emerged as a new approach for chronic wound healing [5]. Neovascularization plays a significant role in wound healing during all stages of the tissue repair process; therefore, a therapy that improves the vascularization of the damaged and regenerating tissue will hasten the healing process, leading to better outcomes [6].

Endothelial progenitor cells (EPCs) are derived from bone marrow and have the potential to differentiate into mature endothelial cells (ECs), leading to tissue vascularization [7]. Velazquez et al. [8] demonstrated that EPCs are present in much higher numbers in non-ischemic wounds compared to ischemic wounds generated in the same animal. This effect is enhanced in diabetic ischemic wounds, where the lack of access to peripheral blood dampens EPC migration to the wound and leads to impaired healing due to poor oxygenation and nutrient and waste transport [9]. This lack of access and impaired EPC recruitment suggest an unmet need for exogenous EPC delivery to ischemic sites. Localized EPC delivery to the ischemic wound site in biological 
scaffolds that support EPC engraftment, angiogenesis and wound healing will have a profound beneficial effect on healing in patients with ischemic wounds.

Porcine small intestinal submucosa (SIS) is an FDA-approved, commercially available, collagen-based natural extracellular matrix (ECM) scaffold that has been widely used clinically for various tissue repairs [10]. SIS serves as a suitable provisional matrix for cell delivery and tissue regeneration because of its good biocompatibility and porous structure [11,12]. SIS scaffolds can also be functionalized in many ways and surface modification has been shown to enormously improve its application in tissue engineering [13]. DS-SILY, is a glycan-based therapy designed to mimic decorin, and is composed of collagen-binding peptides (SILY) conjugated to a dermatan sulfate (DS) backbone [14]. Decorin is a small leucine-rich proteoglycan found abundantly in skin, and is involved in maintaining regularity in collagen fibrillogenesis and preventing hypertrophic scars [15-17]. Similar to the functions of native decorin, a key feature of the DS-SILY molecule is that it binds to collagen matrices, thereby protecting the matrix from extensive and rapid proteolytic breakdown [14]. A further drawback of collagen-rich scaffolds is that they lack sufficient innate biological signals to support sufficient recruitment of EPCs and revascularization of ischemic wounds in vivo. We previously identified LXW7, a cyclic peptide ligand that binds the $\alpha v \beta 3$ integrin receptor on ECs and EPCs, and stimulates cell proliferation and phosphorylation of the VEGF2 receptor [18]. Webber et al. [19] reported that grafting peptides which activate the VEGF receptor to a scaffold results in increased microcirculation and decreased hind-limb ischemia in a mouse model, highlighting the importance of VEGF receptor activation. Hence, the LXW7 peptide ligand could potentially serve to recruit and retain endogenous EPCs by supporting the binding of ECs and EPCs and activating the VEGFR2 receptor through $\alpha v \beta 3$ engagement. In order to assemble a stable and functionalized skin tissue engineering scaffold that can provide a temporary residence for maintaining the angiogenic function of EPCs and support cell survival under ischemic condition, we designed a novel molecule, LXW7-DS-SILY, which is composed of both SILY and LXW7 peptides conjugated to dermatin sulfate. LXW7-DS-SILY binds to the collagen present in the SIS scaffold and also binds to EPCs. Here we demonstrate that when LXW7-DS-SILY is added to SIS the new collagen-rich ECM scaffold increased EPC retention at the ischemic site, enhanced angiogenic function, and ultimately led to wound healing (Figure 1). 


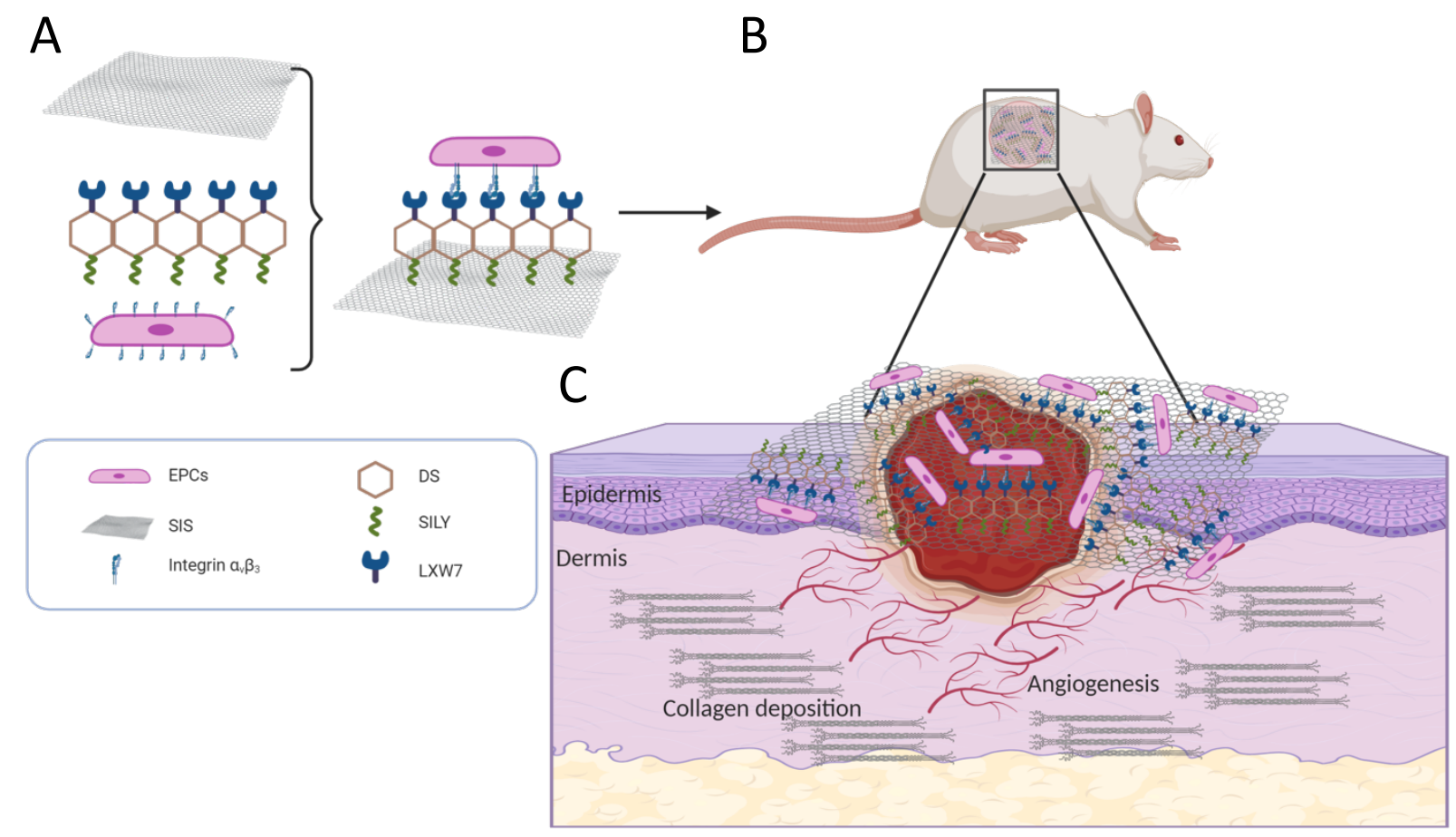

Figure 1 Schematics for the fabrication of ligand modified scaffolds Multiple functional components combined to generate a functionalized scaffold (A) and its topical application to the site of the diabetic ischemic wound of the rat (B) leading to improved vascularization and hastening of the healing of the damaged tissue $(\mathbf{C})$.

\section{Materials and methods}

\subsection{Cell characterization and expansion}

Bone marrow-derived endothelial progenitor cells (EPCs) of Zucker Diabetic Fatty (ZDF) rats were purchased from Cell Biologics, Inc. (RD-6031). EPCs were characterized by DiI-AcLDL staining, immunostaining of CD31 and VE-Cadherin, and tube formation assay. EPCs were cultured in complete rat endothelial cell medium (M1266, Cell Biologics). EPCs were used between passages 5 and 8 for all the experiments described in this study.

\subsubsection{Lentiviral vector transduction}


The lentiviral construct was generated at the University of California Davis Institute for Regenerative Cures (IRC) Vector Core. EPCs were transduced with pCCLc-MNDU3-LUCPGK-Tomato-WPRE lentiviral vector in transduction media consisting of rat basal endothelial cell medium, 5\% FBS, and $8 \mu \mathrm{g} / \mathrm{ml}$ protamine sulfate (MP Biomedicals) at a multiplicity of infection (MOI) of 10 for $6 \mathrm{~h}$. The transduction medium was then replaced with complete rat endothelial cell medium and the cells were cultured for additional $72 \mathrm{~h}$.

\subsubsection{Acetylated low-density lipoprotein uptake assay}

EPCs were cultured in serum-free medium for $12 \mathrm{~h}$ and then incubated with $10 \mu \mathrm{g} / \mathrm{ml}$ DilAc-LDL (Alfa Aesar) for $5 \mathrm{~h}$ at $37{ }^{\circ} \mathrm{C}, 5 \% \mathrm{CO}_{2}$. Cells were then washed three times with Dulbecco's Phosphate buffered saline (DPBS, HyClone) and fixed with 10\% formalin (ThermoFisher Scientific) for $15 \mathrm{~min}$. The nuclei were stained with DAPI (ThermoFisher Scientific) for $5 \mathrm{~min}$. Cells were washed 3 times and imaged with a Zeiss Observer Z1 microscope.

\subsubsection{Tube formation assay}

A 96-well plate was coated with $50 \mu$ Matrigel (354234, Corning) per well and incubated at $37{ }^{\circ} \mathrm{C}, 5 \% \mathrm{CO}_{2}$ to gel for $60 \mathrm{~min} .1 \times 10^{4}$ EPCs were seeded onto the Matrigel-coated wells and incubated at $37{ }^{\circ} \mathrm{C}$ for $16 \mathrm{~h}$. Phase contrast images were taken using Zeiss Observer Z1 microscope.

\subsubsection{Immunofluorescent staining of rat EPCs}

Cells were fixed in 10\% formalin for $10 \mathrm{~min}$ and non-specific binding sites were blocked with $1 \%$ bovine serum albumin (BSA, bioWORLD) in $1 \mathrm{X}$ DPBS for $1 \mathrm{~h}$ at room temperature (RT). The cells were then stained with either CD31 (Abcam, ab119339, 1:200) or VE-Cadherin (ThermoFisher Scientific, 36-1900, 1:200) antibodies in 1\% BSA in DPBS and incubated overnight at $4{ }^{\circ} \mathrm{C}$. Subsequently the cells were then incubated with Alexa488 or Alexa647 conjugated secondary antibodies (ThermoFisher Scientific, 1:500) for $1 \mathrm{~h}$ at RT. The cell nuclei were stained with DAPI for 5 min and imaged using Zeiss Observer Z1 microscope.

\subsection{EPC binding assay on LXW7-beads}


For the on bead cell binding assay, $1 \times 10^{5}$ EPCs were added to an ultralow attachment 24well plate followed by addition of LXW7 resin beads. The plate was placed on shaker set at 40 $\mathrm{rpm}$ and incubated for $15 \mathrm{~min}$ at $37^{\circ} \mathrm{C}, 5 \% \mathrm{CO}_{2}$. Phase contrast images were taken using Zeiss Observer Z1 microscope.

\subsection{Flow cytometry analysis of ligand-cell binding affinity}

To quantitatively determinate ligand-cell binding affinity, flow cytometry analysis of LXW7-biotin (ligand) or D-biotin (negative control) bound to EPCs was performed, as described in our previous study [18]. Briefly, $3 \times 10^{5}$ EPCs were incubated with $1 \mu \mathrm{M}$ LXW7-biotin in a binding buffer (1X HEPES (Gibco) containing 10\% FBS (HyClone) and $1 \mathrm{mM} \mathrm{Mn}^{2+}$ ) on ice for $30 \mathrm{~min}$. The samples were washed three times with wash buffer (DPBS containing 1\% FBS) and incubated with $2 \mu \mathrm{g} / \mathrm{ml}$ of streptavidin-PE-Cy7 conjugate (Life Technologies) on ice for $30 \mathrm{~min}$ and then washed with wash buffer. To test the expression of the $\alpha v \beta 3$ integrin on EPCs, samples were stained with $20 \mu \mathrm{g} / \mathrm{ml}$ mouse anti-rat $\alpha v \beta 3$ integrin antibody (Millipore Sigma) on ice for $30 \mathrm{~min}$, washed three times with wash buffer, and incubated with donkey anti-mouse Alexa647 conjugate (1:500, ThermoFisher Scientific) in DPBS on ice for $30 \mathrm{~min}$ and then washed with DPBS. To confirm that LXW7 binds to EPCs mainly via $\alpha v \beta 3$ integrin, we performed a binding/blocking experiment using a monoclonal anti- $\alpha v \beta 3$ integrin antibody. To block $\alpha v \beta 3$ integrin, cells were first incubated with $20 \mu \mathrm{g} / \mathrm{ml}$ mouse anti-rat $\alpha v \beta 3$ integrin antibody on ice for $30 \mathrm{~min}$, washed three times with wash buffer, and then incubated with LXW7-biotin $(1 \mu \mathrm{M})$ for another $30 \mathrm{~min}$. The samples were washed three times with wash buffer and incubated with 2 $\mu \mathrm{g} / \mathrm{ml}$ Streptavidin-PE-Cy7 conjugate (Life Technologies) in DPBS on ice for $30 \mathrm{~min}$ and then washed with DPBS. Streptavidin-PE-Cy7 only was used as the negative control. Attune NxT Flow Cytometer (ThermoFisher Scientific) was used to perform flow cytometry, and data was analyzed using FlowJo software (FlowJo LLC). The blocking efficiency of LXW7 to ZDF-EPCs was calculated according to the following formula: blocking efficiency of LXW7 to ZDF-EPCs $=(\mathrm{B} 0-\mathrm{Bb}) / \mathrm{B} 0 * 100$, where $\mathrm{B} 0$ is designated as the initial ZDF-EPC-LXW7 binding percentage and $\mathrm{Bb}$ is designated as the ZDF-EPC-LXW7 binding percentage after ZDF-EPCs were blocked with anti- $\alpha v \beta 3$ integrin antibody.

2.4 Synthesis and characterization of peptide-hydrazides 
Hydrazide-modified peptides RRANAALKAGELYKSILYGSG-hydrazide (SILY-hydrazide) and cGRGDdvc(AEEA) ${ }_{2}$ WG-hydrazide (LXW7-hydrazide, wherein AEEA is short PEG linker) were synthesized using standard Fmoc solid-phase peptide synthesis according to a previously published protocol [20, 21]. Briefly, Cl-TCP(Cl) ProTide Resin (loading 0.4-0.6 mmol/g, CEM Corporation) was rinsed 3 times with dichloromethane (DCM, Fisher Scientific) and N, NDimethylformamide (DMF, Fisher Scientific) and swollen in 50\% DCM/DMF for $1 \mathrm{~h}$. Swollen resin was then reacted twice with 10\% hydrazine hydrate (Sigma) in DMF and 0.057 M N, NDiisopropylethylamine (DIPEA, Fisher Scientific) for $2 \mathrm{~h}$ at RT each. 10\% methanol (Fisher Scientific) in DMF was used to cap any unreacted chloride groups, and the resin was washed 3 times with DMF and 3 times with DCM. The resin was then reacted with 4 equivalents of the first Fmoc-amino acid with 4 equivalents of OxymaPure, N, $N^{\prime}$-Diisopropylcarbodiimide (DIC, Fisher Scientific) and 10 equivalents of DIPEA in DMF for $4 \mathrm{~h}$, followed by washing three times with DMF and three times with DCM. Subsequent amino acids were coupled for 10 min each at $50{ }^{\circ} \mathrm{C}$ on a Liberty Blue microwave peptide synthesizer (CEM Corporation) using 5 equivalents of Fmoc-amino acids, DIC, and OxymaPure with 0.1M DIPEA. 20\% piperidine in DMF was used for deprotection. Peptides were cleaved off beads using $88 \%$ trifluoroacetic acid (TFA, Fisher Scientific), 5\% phenol (Sigma), 5\% H2O, and 2\% Triisopropylsilane (TIPS, Sigma) for $3 \mathrm{~h}$. Crude peptides were precipitated in cold diethylether (Acro organics) and allowed to dry before dissolving in $5 \%$ acetonitrile/ $\mathrm{H}_{2} \mathrm{O}$ for purification. Before purifying, LXW7 was cyclized by oxidizing cysteine residues using ClearOx resin (Peptides International) according to manufacturer's protocol. Peptides were purified through a C18 prep column against an acetonitrile gradient on an AKTApure 25 FPLC (GE Healthcare) and confirmed by MALDITOF-MS (Bruker). For some experiments, peptides were purchased from InnoPep.

2.5 Synthesis and characterization of molecule variants

DS-SILY or LXW7-DS-SILY were prepared by conjugating SILY-hydrazide and LXW7hydrazide to a dermatan sulfate (DS) backbone using carbodiimide chemistry. DS (average molecular weight 41,816 Da, Celsus Laboratories) was reacted with peptide-hydrazides using 1ethyl-3-[3-dimethylaminopropyl] carbodiimide hydrochloride (EDC, ThermoFisher Scientific) in 0.1 M MES [2-(N-morpholino)ethanesulfonic acid] buffer with $8 \mathrm{M}$ urea (Sigma) and 0.6\% $\mathrm{NaCl}$ (Sigma) titrated to $\mathrm{pH}$ 4.5. First, SILY-hydrazide was conjugated to the DS for $4 \mathrm{~h}$ using 
$0.01 \mathrm{mM}$ EDC. The reaction was stopped by titrating the $\mathrm{pH}$ to 8 . The product was purified using tangential flow filtration (Spectrum labs) with a 10kDa column and then lyophilized. LXW7hydrazide was then sequentially conjugated to the DS-SILY construct in a similar manner using $0.1 \mathrm{mM}$ EDC for $24 \mathrm{~h}$ before purification. Peptide conjugation was verified by creating standard curves for SILY and LXW7 using concentration dependent $280 \mathrm{~nm}$ absorbance of aromatic amino acids and extrapolating absorbances of synthesized molecules using readings taken on a NanoDrop UV-Vis spectrophotometer (Thermo Fisher). The structures of LXW7-DS-SILY and the reaction scheme of making LXW7-DS-SILY was described in a previous study [22].

2.6 Preparation of ligand-modified SIS scaffolds with or without EPCs.

8-mm diameter punch-outs of SIS-ECM (Cook Biotech) were cut using a sterile biopsy punch and placed into wells of a 48-well plate with the rough side facing up and incubated with $0.2 \mathrm{ml}$ of $10 \mu \mathrm{M}$ LXW7-DS-SILY, DS-SILY or DPBS for $1 \mathrm{~h}$ at $37{ }^{\circ} \mathrm{C}$. Scaffolds were subsequently rinsed with DPBS for three times and then soaked overnight in complete culture media at $37{ }^{\circ} \mathrm{C}, 5 \% \mathrm{CO}_{2} .5 \times 10^{5} \mathrm{Td}$-Tomato/luciferin-labeled EPCs were resuspended in $15 \mu 1$ of complete media per ECM and carefully added onto the surface of LXW7-DS-SILY, DS-SILY ligand modified or unmodified SIS. The plate was placed in a $37{ }^{\circ} \mathrm{C}, 5 \% \mathrm{CO}_{2}$ incubator and incubated for $1 \mathrm{~h}$ to allow for cell adherence before addition of $0.3 \mathrm{ml}$ of complete media per well.

\subsection{Attachment assay and CCK-8 assay}

To modify the culture surface with ligands, plates were coated with $200 \mu \mathrm{l}$ of $20 \mu \mathrm{g} / \mathrm{ml}$ Avidin (Thermo Fisher) and incubated for $1 \mathrm{~h}$ at $37^{\circ} \mathrm{C}$. Avidin coated wells were rinsed three times with DPBS and were treated with $200 \mu$ molar equivalents $(2 \mu \mathrm{M})$ of LXW7-biotin or Dbiotin (negative control). The wells were washed three times with DPBS in $1 \mathrm{~h}$ and blocked with $1 \%$ BSA for $1 \mathrm{~h}$. After the wells were rinsed three times with DPBS, $2 \times 10^{4}$ EPCs were added to the wells and incubated for $20 \mathrm{~min}$ at $37^{\circ} \mathrm{C}$ and $5 \% \mathrm{CO} 2$. To further explore the effect of LXW7 to the binding of EPCs on an ECM scaffold surface, LXW7-DS-SILY modified SIS and untreated control SIS were placed in an ultralow attachment 48 -well plate. The scaffolds were rinsed with DPBS and seeded with EPCs at a density of $1.5 \times 10^{5}$ cells $/ \mathrm{cm}^{2}$. After 20 min of incubation, unattached cells were washed off with DPBS three times. Images were taken using 
Zeiss Observer Z1 microscope. Three independent experiments were performed. Cell counter ImageJ plugin was used to determine the cell numbers in three randomly selected fields from each independent experiment.

The effect of LXW7 on the EPC growth was determined by a Cell Counting Kit-8 assay (CCK-8, Dojindo). This assay was used to detect cell viability. The 96-well plates were treated with $1 \mu \mathrm{M}$ Avidin solution for $1 \mathrm{~h}$ at RT then rinsed three times with DPBS. Wells were treated with $1 \mu \mathrm{M}$ LXW7-biotin (ligand) or $1 \mu \mathrm{M}$ D-biotin (negative control) for $1 \mathrm{~h}$, washed three times with DPBS and blocked with $1 \%$ BSA for $1 \mathrm{~h}$. A total of $1.5 \times 10^{3}$ viable EPCs were seeded per well of the 96-well plate and cultured for 5 days. To test the growth of EPCs on LXW7-DS-SILY modified SIS scaffolds, we prepared the ligand modified scaffolds, as described above in section 2.6. A total of $5 \times 10^{4}$ viable EPCs were seeded per scaffold and cultured for 5 days. The CCK-8 assay was performed according to the manufacturer's instructions. Absorbance was measured at $450 \mathrm{~nm}$ using a SpectraMax i3 plate reader instrument (Molecular Devices LLC).

2.8 Cell loading density

The optimal loading density of EPCs on SIS-ECM was determined by the CCK-8 assay. EPCs were seeded on 8-mm SIS-ECM discs at the following densities: $0,4.2 \times 10^{4}, 1 \times 10^{5}, 3 \times$ $10^{5}, 5 \times 10^{5}$, and $1 \times 10^{6}$ cells $/ \mathrm{cm}^{2}$. At $24 \mathrm{~h}$ after seeding, absorbance was measured at $450 \mathrm{~nm}$.

\subsection{Zucker Diabetic Fatty rat ischemic skin wound model}

All animal procedures were approved by The University of California, Davis (UCD) Institutional Animal Care and Use Committee (IACUC). The ligand-modified SIS scaffolds loaded with or without EPCs were prepared as described above in methods section 2.6. Male Zucker Diabetic Fatty rats (ZDF fa/fa, Charles River, 12 to 16 weeks old) were used and placed on a special diet to induce Type II diabetes (Purina \#5008 from Lab Diet, Inc.). The blood glucose levels of ZDF rats in each group were measured using a digital glucose meter and test strips (Accu-Chek ${ }^{\circledR}$ Sensor, Roche Inc., Mannheim, Germany) 2 or 3 days prior to surgery and at the study endpoint. The rat ischemic skin wound model was carried out as previously described with some modifications [23]. Briefly, animals were placed under isoflurane anesthesia (5\% induction) and maintained (1-3\%) on a non-rebreathable circuit with charcoal filter scavenging. After shaving and sterilization, two 6-mm diameter full-thickness ischemic wounds were created 
using a biopsy punch in the center of the designated flap area (Figure $\mathrm{S} 1,10 \mathrm{~cm} \times 3 \mathrm{~cm}$ ). Incisions were then made along the marked lines down to the paraspinous muscle to create a bipedicle flap and separate the panniculus carnosus fascia from the paraspinous muscles. A sheet of sterile silicone was placed underneath to induce ischemia. 5-0 non-absorbable sutures (J494G, eSutures) were used to close both incisions by anchoring the silicone sheet to the skin with multiple interrupted stitches along the length of the flap. Lastly, two 6-mm diameter fullthickness non-ischemic wounds were created using a biopsy punch lateral to the bipedicle flap on either side of the flap. The wounds created were then covered with the following SIS-scaffold treatment groups: SIS only, SIS/DS-SILY, SIS/LXW7-DS-SILY, SIS/EPCs, SIS/DS-SILY/EPCs, or SIS/LXW7-DS-SILY/EPCs (prepared as described in section 2.6). The EPCs-loaded SIS scaffolds were placed with cells in direct contact with the site of the wound. Scaffolds were immobilized with 4 stitches using 5-0 silk sutures (VP710X, eSutures). To protect the wound, a Tegaderm transparent dressing (3M Health Care, Germany) was then applied to all wounds. At days $0,3,7,11$, and 14 post-treatment, pictures were taken of all wounds, blinded for treatment type. The wound area was calculated by tracing the wound margins from rats and was evaluated as a percent area of the original wound using Image-Pro Plus 6.0 software. The percentage of wound reduction was calculated according to the following formula. Rate of wound closure $=$ $(\mathrm{A} 0-\mathrm{At}) / \mathrm{A} 0 * 100$, where $\mathrm{A} 0$ and At are designated as the initial wound area and wound area at the designated time, respectively.
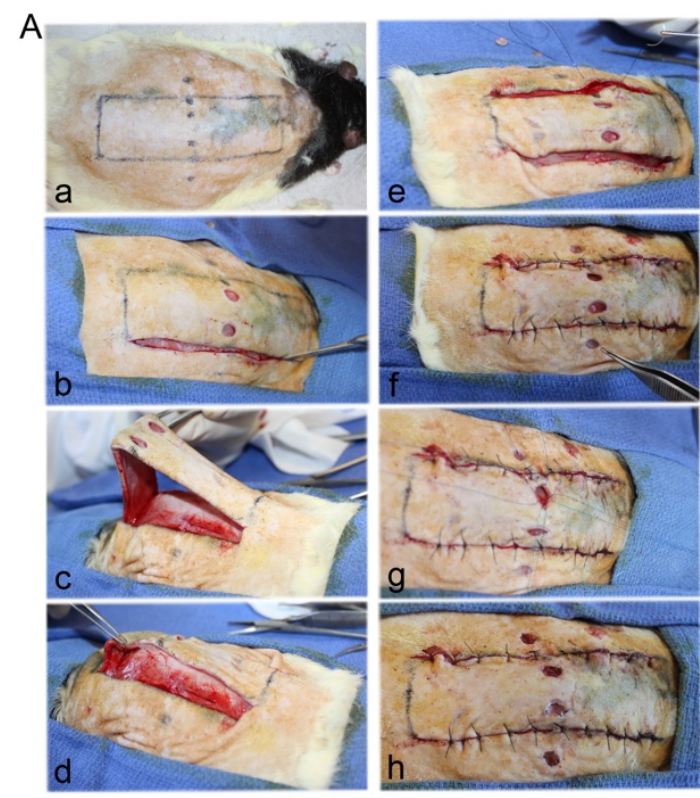

B

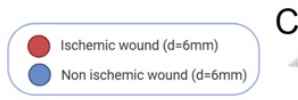

C
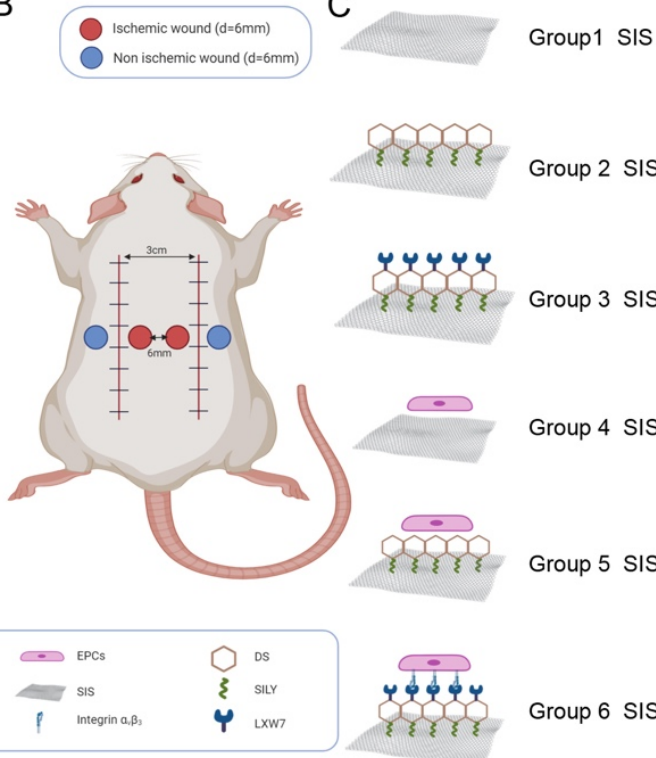

Group 2 SIS/DS-SILY

Group 3 SIS/LXW7-DS-SILY

Group 4 SIS/EPCs

Group 5 SIS/DS-SILY/EPCS

Group 6 SIS/LXW7-DS-SILY/EPCs 


\section{Figure S1 Ischemic skin flap model of Zucker Diabetic Fatty rat and experimental design}

A-B. Demonstrates the process of making Zucker Diabetic fatty rat ischemic skin wounds model.

C. Demonstrates the different ligand modified SIS groups used to treat the wounds.

\subsection{Bioluminescence imaging}

Scaffolds seeded with EPCs in the ZDF rats were monitored via In Vivo Imaging Spectrum (IVIS) system (PerkinElmer) at designated time points (1, 3, 7 and 11 days post-treatment). Prior to imaging, the rats were weighed, and luciferase substrate D-luciferin (Gold Biotechnology) was injected subcutaneously into the animals at $150 \mathrm{mg} / \mathrm{kg}$ body weight. Five min post-injection, the rats were anesthetized using 2\% isoflurane for $5 \mathrm{~min}$ and imaged under anesthesia. Images were analyzed using Living Image ${ }^{\circledR} 2.50$ (Perkin Elmer). The bioluminescence signal intensity was represented as total photons of the region of interest (ROI) subtracted by the region of no positive signal in the same animal.

\subsection{Histological analysis}

Animals were euthanized on day 14 and their wound tissues were carefully biopsied. Samples were fixed with $4 \%$ paraformaldehyde for $24 \mathrm{~h}$, protected by $30 \%$ sucrose dehydration for $48 \mathrm{~h}$, and embedded in O.C.T compound (Sakura Finetek USA). Sections (10 $\mu \mathrm{m}$ thick) were cut using a Cryostat (Leica CM3050S) and stained with Hematoxylin and Eosin (H\&E) to visualize tissue formation or Masson trichrome staining to observe collagen deposition during the healing period. A total of 24 single images were captured at 10X magnification, merged and analyzed using a Keyence BZ9000 microscope and the BZ II Analyzer software. A total of 5 sections from 5 rats of each group were analyzed.

For immunofluorescence staining, tissue sections were washed with DPBS, blocked with 5\% BSA for $1 \mathrm{~h}$ in DPBS, and stained with the following primary antibodies by incubating at $4{ }^{\circ} \mathrm{C}$ overnight: RECA-1 (1:50, ab9774, mouse, Abcam), $\alpha$-SMA (1:200, ab5694, rabbit, Abcam), Collagen I (1:200, ab21286, rabbit, Abcam), Collagen III (1:200, ab7778, rabbit, Abcam). Sections were then incubated with their respective secondary antibodies diluted at 1:500 for $1 \mathrm{~h}$. The slides were counterstained with DAPI $(1: 5,000)$ for $5 \mathrm{~min}$ and mounted with Prolong Diamond Antifade Mountant (Invitrogen). A Nikon A1 laser-scanning confocal microscope was 
used to acquire confocal images. The number of blood vessels (RECA positive) per field were counted and mean fluorescence intensity were measured by Image J. A total of 6 sections from 3 rats of each group were analyzed.

\subsection{Statistics}

Data are reported as mean \pm standard deviation (SD) for bead-binding, block efficiency, cell attachment, CCK-8 assay and cell loading density assay and as mean \pm standard error of mean (SEM) for healing rate, bioluminescence images and histological analysis. Statistical analysis of beads binding assay and cell attachment assay was performed using the Student's t-test. Analyses of bioluminescence images, histological analysis were performed using one-way ANOVA. Healing rate analysis, CCK-8 assay was performed by two-way ANOVA. All statistical analyses were performed using PRISM 7 (GraphPad Software Inc.), and differences were considered significant when $\mathrm{p}<0.05$.

\section{Results}

\subsection{Characterization and transduction of ZDF rat bone marrow EPCs}

ZDF rat bone marrow EPCs (ZDF-EPCs) displayed the typical morphology of EPC colonies (Figure S2A) and were efficiently transduced with lentiviral vector expressing a fluorescent marker Td-tomato for tracking analysis (Figure S2B). Strong expression of EPC markers (CD31 and VE-cadherin) confirmed their EC characteristics (Figure S2C-D). The tube formation assay results revealed their ability to assemble into tubules in the presence of a basement membrane in vitro (Figure S2E). After co-culturing with DiI-Ac-LDL, positive staining for DiI-Ac-LDL was observed in ZDF-EPCs (Figure S2F). These results indicated that ZDFEPCs have similar phenotype and function as human EPCs. 


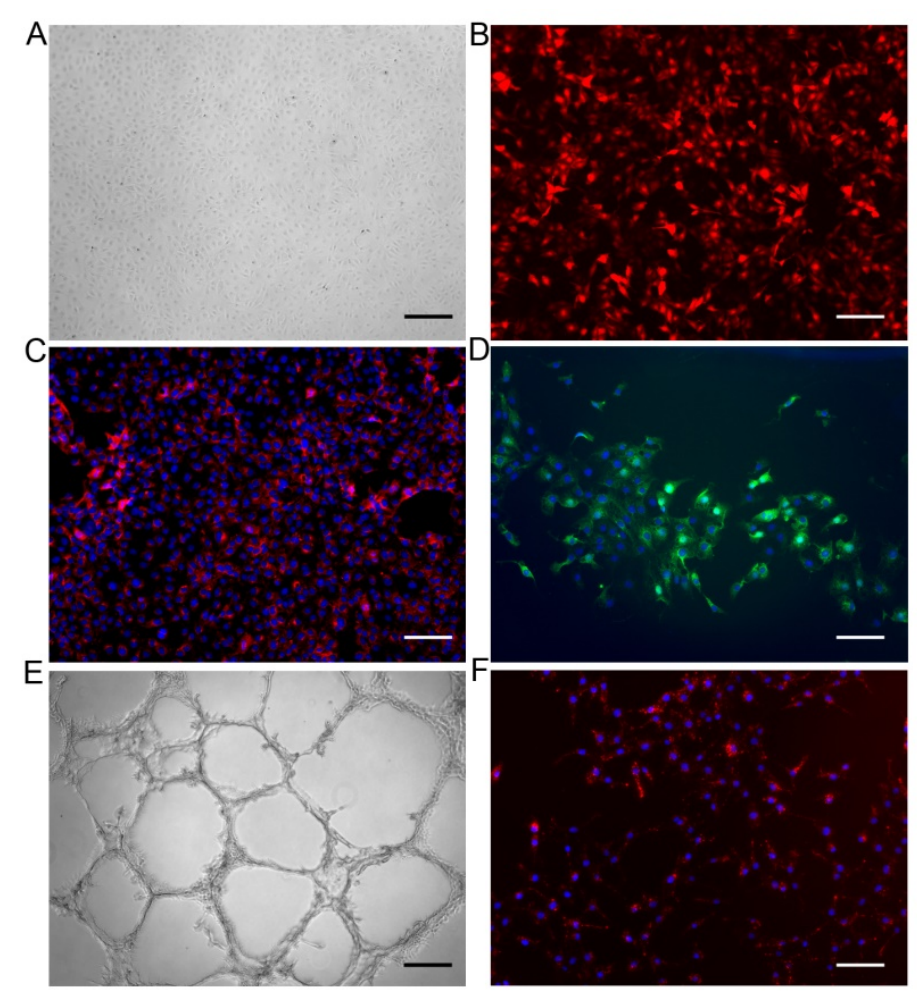

Figure S2 Characterization of ZDF-EPCs. A-B. Representative phase contrast (A) and fluorescent (B) images of pCCLc-MNDU3-LUC-PGK-Tomato-WPRE lentiviral vector transduced ZDF-EPCs. C-D. Immunofluorescence staining results of expression of CD31 (C) and VE-Cadherin (D). E. In vitro tube formation results of ZDF-EPCs. F. Acetylated low-density lipoprotein uptake by ZDF-EPCs. Scale bar $=100 \mu \mathrm{m}(\mathrm{C}, \mathrm{D}, \mathrm{F})$. Scale bar $=200 \mu \mathrm{m}(\mathrm{A}, \mathrm{B}, \mathrm{E})$.

\subsection{LXW7 ligand showed excellent binding affinity to ZDF-EPCs via the $\alpha v \beta 3$ integrin}

In our previous studies, we identified LXW7 as a potent ligand that specifically targets EPCs and ECs normally present in circulation via binding to $\alpha v \beta 3$ integrin on cell surfaces [18, 24]. To confirm the binding of LXW7 to the ZDF-EPCs, resin beads displaying LXW7 were incubated with ZDF-EPCs. THP-1 monocytes served as a negative control. After 30 min of incubation, the LXW7 beads showed strong binding to ZDF-EPCs but not to THP-1 monocytes (Figure 2A). Quantification of the number of cells bound on each bead showed that there was a significant increase in the binding of ZDF-EPCs to LXW7 resin beads compared to THP-1 monocytes (Figure 2B). To further confirm that LXW7 binds to ZDF-EPCs mainly via $\alpha v \beta 3$ integrin, we performed flow cytometry analysis to detect the expression of $\alpha v \beta 3$ integrin and 
performed a binding/blocking experiment using a monoclonal anti- $\alpha v \beta 3$ integrin antibody. The results showed $\alpha v \beta 3$ integrin was expressed on the majority of ZDF-EPCs [(75.33 $\pm 4.25 \%$ of overall populations) Figure 2C]. From the binding/blocking experiment, as shown in Figure 2D, we confirmed that LXW7 had high binding efficiency to ZDF-EPCs, and LXW7 binding to ZDF-EPCs was blocked by the anti- $\alpha v \beta 3$ integrin antibody (Figure 2D). The average blocking efficiency was $60.8 \pm 22.1 \%$ (Figure 2D). These data confirmed that the binding of LXW7 to ZDF-EPCs was primarily mediated via $\alpha v \beta 3$ integrin.

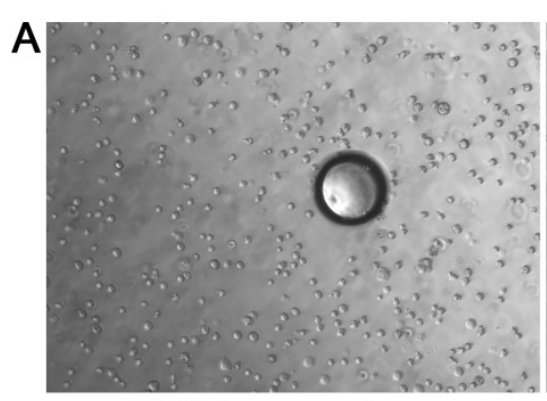

THP-1 monocytes

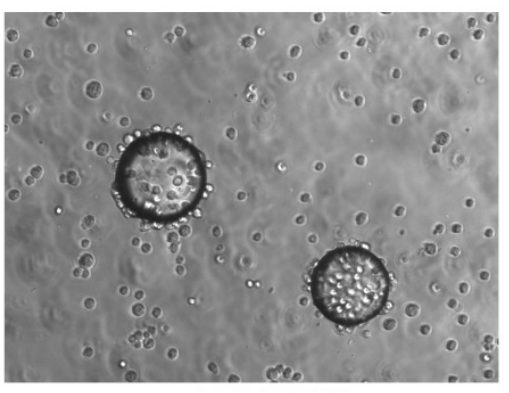

ZDF EPCs
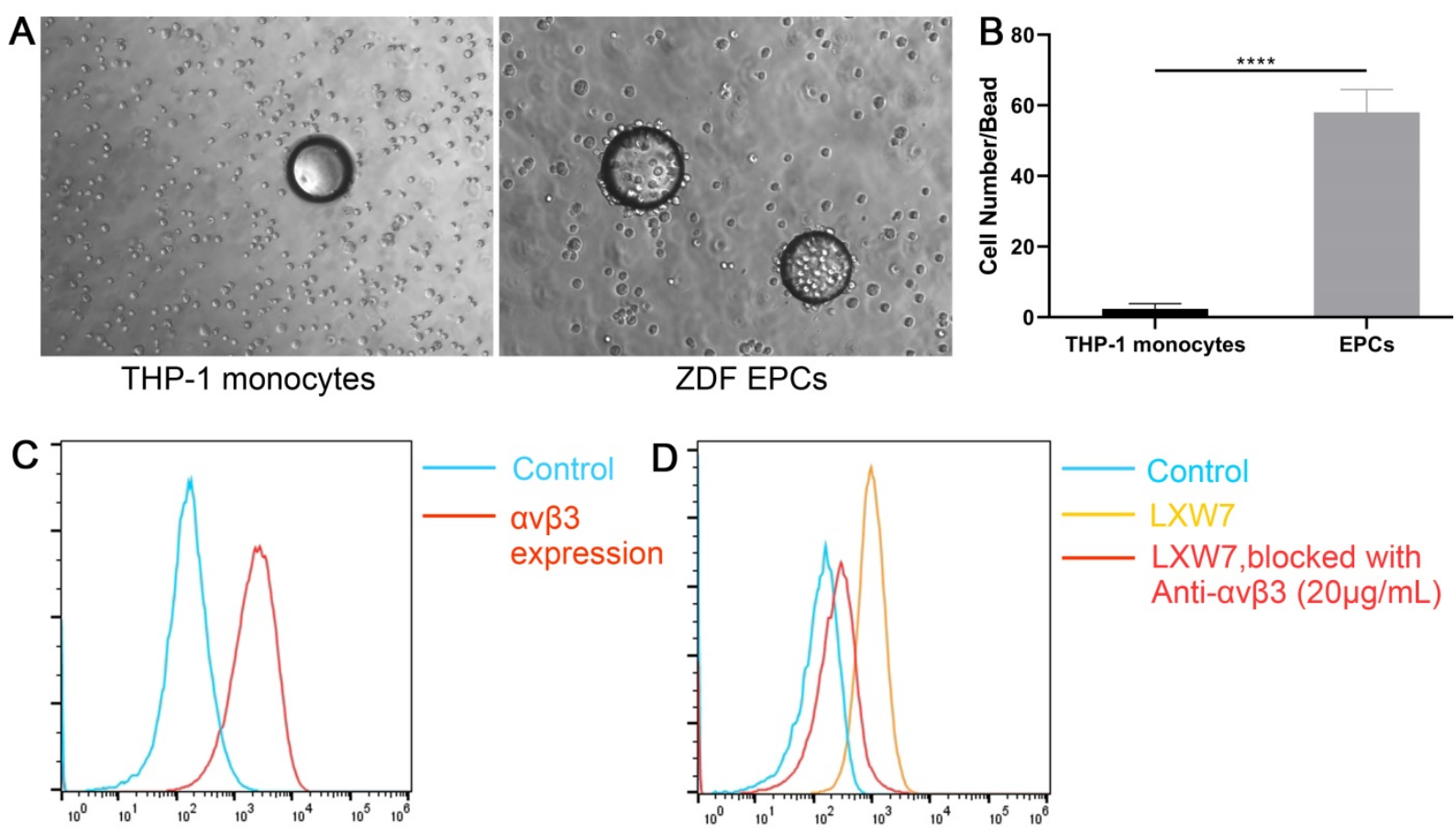

Fig.2 Specificity and binding affinity of LXW7 ligand to ZDF-EPCs and expression of $\alpha \mathrm{v} \beta 3$ integrin on ZDF-EPCs

A. On-bead cell binding assay for testing the binding affinity and specificity of ligands to ZDFEPCs showing high binding of ZDF-EPCs to LXW7 coated beads (right panel) compared to THP-1 monocytes (left panel). B. Quantification of the number of THP-1 monocytes and ZDFEPCs bound to the LXW7 coated beads. C. Expression of $\alpha v \beta 3$ integrin on ZDF-EPCs. D. Flow cytometry analysis of the binding affinity of LXW7 showing the blocking of its binding by anti$\alpha v \beta 3$ antibody. Scale bar $=100 \mu \mathrm{m}$. Data are expressed as mean \pm SD. $* * * * p<0.0001, n=3$.

3.3 LXW7 modified surfaces increased ZDF-EPC attachment and growth 
To determine if the LXW7 ligand can support effective ZDF-EPC attachment and growth, we employed both 2D tissue culture surface and 3D ECM-based scaffolds to determine cellligand binding ability in different situations. We treated tissue culture polystyrene with LXW7biotin (ligand) or D-biotin (negative control) and investigated selective attachment of ZDF-EPCs. To further explore the binding ability of LXW7 to ZDF-EPCs on ECM-based scaffolds, we incubated LXW7-DS-SILY with SIS to allow binding of the biofunctional ligand to the SIS. Seeding ZDF-EPCs on both the 2D and 3D surfaces showed that LXW7 and LXW7-DS-SILY can support attachment of ZDF-EPCs both to plate and SIS surfaces (Figure 3A, 3B, 3D, 3E). The number of cells attached was quantified and the results demonstrated that the LXW7-treated surfaces attracted more ZDF-EPCs than the unmodified surfaces (Figure 3C, 3F respectively).

The effect of LXW7 on the growth of ZDF-EPCs was tested by CCK-8 assay at different time points in a course of 5 days. Results showed that the LXW7-biotin treated surface significantly promoted the growth of ZDF-EPCs after $48 \mathrm{~h}$ in culture compared with the D-biotin treated surface (control), and this trend was maintained for the entire period of the experiment (Figure 3G). Similar to the trend on tissue culture plates, SIS/LXW7-DS-SILY also promoted the growth of ZDF-EPCs when compared to SIS/DS-SILY and SIS (Figure 3H). These results demonstrate that the LXW7-modified surface and functionalized scaffold support attachment and growth of EPCs. 

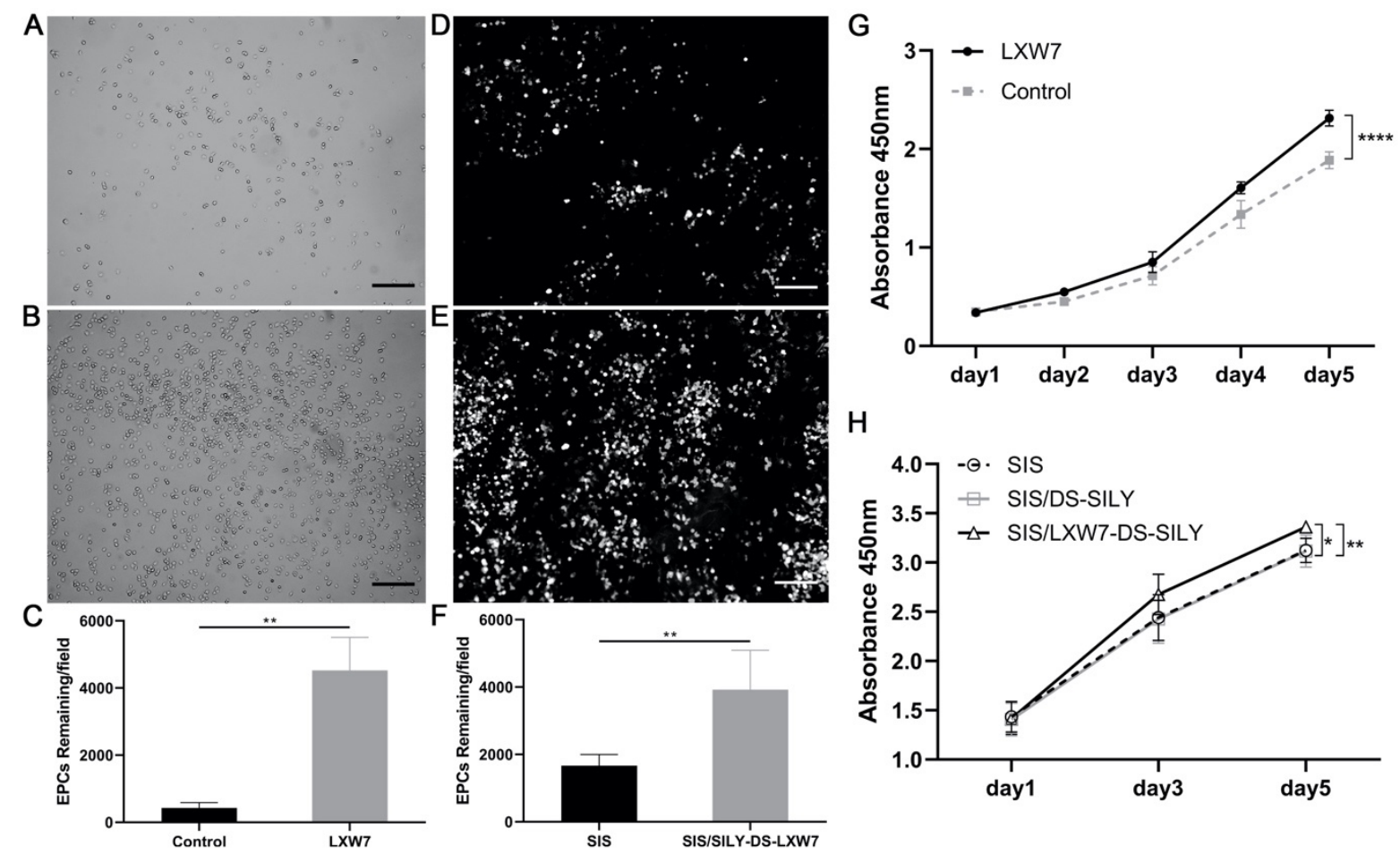

$\mathrm{H}$

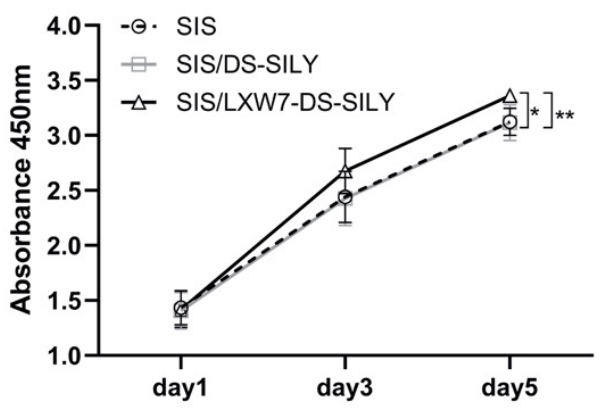

Figure 3 Effect of LXW7 and LXW7-DS-SILY ligands on the attachment, growth and viability of ZDF-EPCs on ligand modified surfaces.

A-B. Representative images of attached ZDF-EPCs on surfaces treated by D-biotin (A; control), LXW7 after 20 min incubation. (C). Quantification and the correlative statistical analysis of remaining cells shown in A-B. D-E. Representative images of adhered EPCs on untreated (D; control) or LXW7-DS-SILY modified SIS surface (E) after 20 min incubation. F. Quantification and the correlative statistical analysis of remaining cells shown in D-E. G. Growth and viability of ZDF-EPCs on LXW7 treated tissue culture surfaces and D-biotin treated surfaces (control) was assessed by CCK-8 assay. H. Growth and viability of ZDF-EPCs on LXW7-DS-SILY or DS-SILY treated SIS scaffold surfaces was assessed by CCK-8 assay. Scale bar $=200 \mu \mathrm{m}$. Data are expressed as mean $\pm \mathrm{SD} . * * * * \mathrm{p}<0.0001, * * \mathrm{p}<0.01, * \mathrm{p}<0.05, \mathrm{n}=3$.

\subsection{In vivo wound healing studies}

A bipedicle cutaneous flap was created on each rat to yield 2 ischemic and 2 non-ischemic wounds per animal (Figure S1A, S1B). Both ischemic and non-ischemic wounds were implanted with ligand-modified or unmodified SIS patches seeded with or without ZDF-EPCs at a seeding density of $5 \times 10^{5}$ cells $/ \mathrm{cm}^{2}$. This seeding density was based on the result obtained from our 
CCK-8 assay where a decrease in cell viability was observed beyond a density of $5 \times 10^{5}$ cells $/ \mathrm{cm}^{2}$ (Figure S3). The average blood glucose level of ZDF rats in each group $>250 \mathrm{mg} / \mathrm{dl}$.

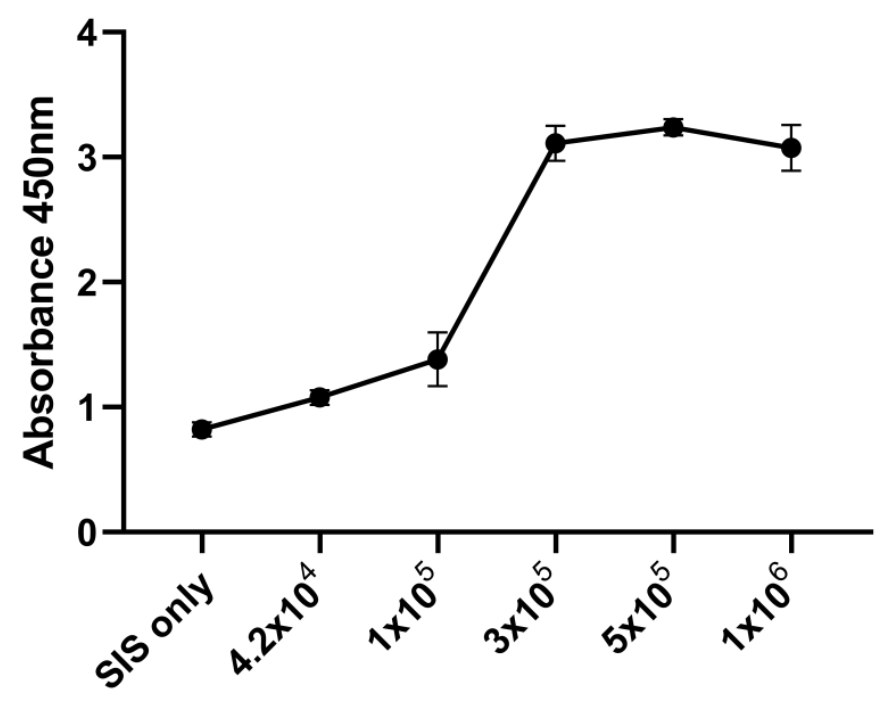

Figure S3 ZDF-EPCs loading density on SIS-ECM. Mean results of CCK-8 loading assay demonstrates the absorbance values of increasing concentrations of ZDF-EPCs seeded on SISECM up to $5 \times 10^{5}$ cells $/ \mathrm{cm}^{2} . \mathrm{n}=3$.

3.4.1 LXW7-DS-SILY modified SIS scaffolds accelerated diabetic non-ischemic wound healing and supported survival of ZDF-EPCs

The diabetic wound healing assay was performed to evaluate the effect of the different scaffolds on non-ischemic wound healing in vivo. The wound regions of the diabetic rat were treated with SIS/LXW7-DS-SILY/EPCs, SIS/DS-SILY/EPCs, SIS/EPCs, SIS/LXW7-DS-SILY, SIS/DS-SILY and SIS respectively. Digital photographs were obtained 0, 3, 7, 11, and 14 days after surgery (Figure 4A). In the absence of exogenous ZDF-EPC seeding, the wound area in all groups decreased over time, and the average observed wound closure rate was significantly increased in the SIS/LXW7-DS-SILY group $(80.7 \pm 0.03 \%)$ compared to both the SIS/DS-SILY $(56.9 \pm 0.01 \%)$ and SIS $(56.3 \pm 0.05 \%)$ groups on day 14 (Figure 4B panel a). Among all groups loaded with exogenous ZDF-EPCs, the group treated with SIS/LXW7-DS-SILY/EPCs showed a wound healing rate of $82.5 \pm 0.02 \%$, significantly higher than that of SIS/DS-SILY/EPCs $(69.7 \pm 0.03 \%)$ and SIS/EPCs $(67.2 \pm 0.03 \%)$ groups (Figure 4B panel b). However, in the non- 
ischemic wounds, ZDF-EPCs did not accelerate wound closure when comparing SIS/LXW7-DSSILY/EPCs with SIS/LXW7-DS-SILY or comparing SIS/EPCs with SIS. When compared to SIS/DS-SILY, SIS/DS-SILY/EPCs accelerated wound healing. (Figure 4B panels a-e).

To investigate cell survival of transplanted cells in ZDF rats, Td-Tomato/luciferin-labeled ZDF-EPCs were seeded on different scaffolds and monitored by IVIS. The bioluminescence signal in the SIS/LXW7-DS-SILY/EPCs group showed a significantly higher intensity when compared to SIS/DS-SILY/EPCs and SIS/EPCs groups at day 1, 3 and 7, suggesting that LXW7 improved ZDF-EPC retention in the wound bed at early time points (Figure 4C). The intensity in all the three groups decreased to baseline by day 11 (Figure 4D). 

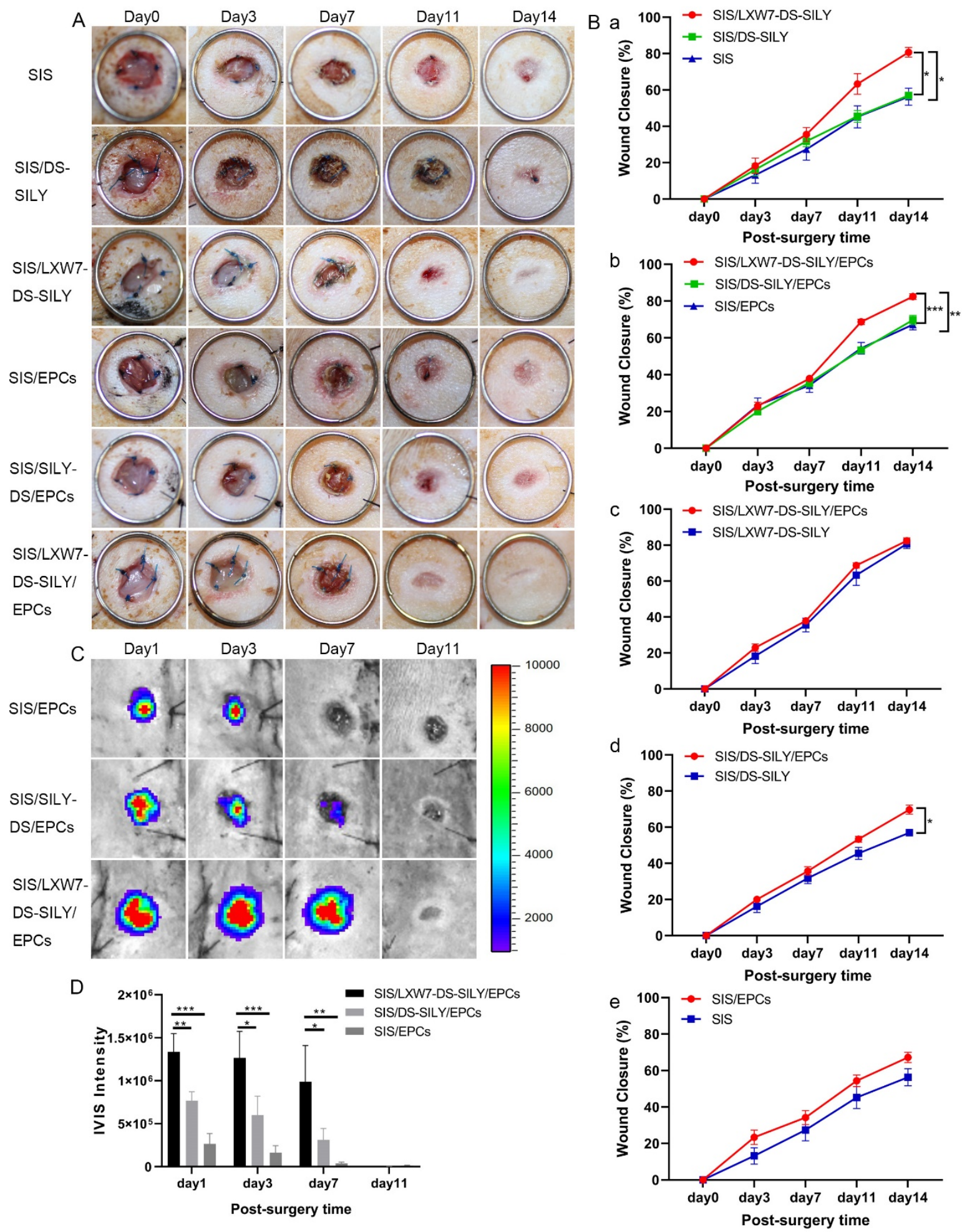

Figure 4 SIS/LXW7-DS-SILY scaffolds accelerate wound closure and support ZDF-EPC survival under non-ischemic condition.

A. Representative images of healing in wounds treated with different groups at day $0,3,7,11$ 
and 14. B. Quantification of the healing rate of different groups. Data are expressed as mean \pm SEM. SIS, SIS/LXW7-DS-SILY, SIS/EPCs group: n=8; SIS/DS-SILY: n=7; SIS/DS-SILY/EPCs: $\mathrm{n}=12$; SIS/LXW7-DS-SILY/EPCs: $\mathrm{n}=13$. C. Bioluminescence imaging of wounds transplanted with different modified SIS scaffolds loaded with Td-Tomato/luciferin-labeled ZDF-EPCs. D. Quantification of the signal intensity of bioluminescence (Most of the SIS scaffolds spontaneously fall off the wounds by day 11). Data are expressed as mean \pm SEM. $n=5$ per group. $* * * \mathrm{p}<0.001, * * \mathrm{p}<0.01,{ }^{*} \mathrm{p}<0.05$.

3.4.2 LXW7-DS-SILY modified SIS scaffolds accelerated wound healing and re-epithelialization in non-ischemic wound areas.

To further compare the healing quality of wounds treated by different scaffolds, H\&E staining was performed on non-ischemic wound tissue samples (Figure 5A). As shown in Figure 5C, the SIS/LXW7-DS-SILY/EPCs group showed the shortest residual wound length among all the groups, followed by SIS/LXW7-DS-SILY, SIS/DS-SILY/EPCs, SIS/EPCs, SIS and SIS/DSSILY groups at day 14. Also, the SIS/LXW7-DS-SILY/EPCs and SIS/DS-SILY/EPCs groups were fully covered with neoepidermis, while in the other groups, re-epithelialization was not fully completed (Figure 5D). Overall, the SIS/LXW7-DS-SILY/EPCs group showed a higher efficacy of wound healing in comparison with other groups. 

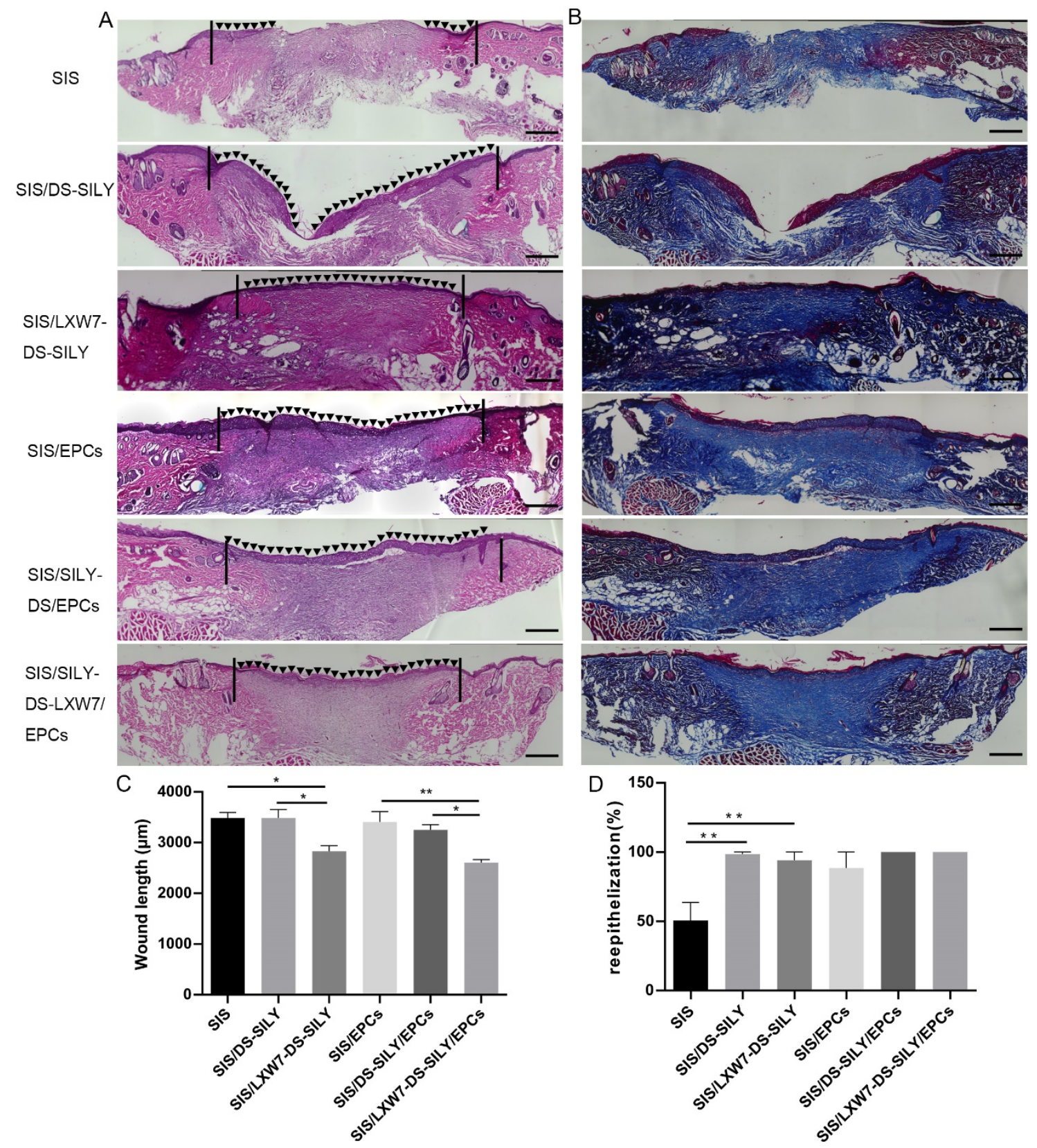

Figure 5 A. Representative images of H\&E staining of wounds at day 14 under non-ischemic condition. B. Representative images of Masson Trichrome staining of wounds at day 14 C-D. Quantification of the wound length (C) and percentage of re-epithelization (D) of different modified SIS scaffolds. Scale bar $=500 \mu \mathrm{m}$. Data are expressed as mean \pm SEM. $* * p<0.01$, $* \mathrm{p}<0.05, \mathrm{n}=5$ per group. 
3.4.3 LXW7-DS-SILY modified SIS scaffolds promoted neovascularization and stimulated collagen deposition in non-ischemic wound areas

To evaluate neovascularization, we morphometrically assessed blood vessel density at wound sites using immunostaining for rat anti-endothelial cell antibody-1 (RECA-1) and alphasmooth muscle actin ( $\alpha$-SMA) at day 14 (Figure 6A). Wounds treated with SIS/LXW7-DS-SILY scaffold showed a significantly higher vessel number than SIS/DS-SILY and SIS scaffolds in the regenerated tissue (Figure 6D). When wounds were treated with different scaffolds seeded with ZDF-EPCs, SIS/LXW7-DS-SILY/EPCs promoted neovascularization compared to control scaffolds with ZDF-EPCs (Figure 6D). In addition, blood vessels in the SIS/LXW7-DSSILY/EPCs group displayed a mature structure with a larger lumen when compared to control groups (Figure 6A).

To observe collagen morphology and distribution, Masson Trichrome staining was conducted. Masson staining showed arrangement of newly formed collagen in the regenerated tissue in all six groups (Figure 5B). On day 14, densely packed and basket-weave patterns typical of collagen fibers were observed for the SIS/LXW7-DS-SILY/EPCs group while only sparse, loosely packed collagen fibers were observed in the SIS-only group (Figure 5B)

To detect whether the collagen deposition could be stimulated by the various treatment groups, collagen immunostaining of the tissue sections was performed. Enhanced collagen I and collagen III staining intensity was observed in SIS/LXW7-DS-SILY/EPCs treated wounds on day 14, compared to the other wounds (Figure 6B, 6C). SIS/LXW7-DS-SILY also showed a significantly higher collagen I and III staining intensity compared to SIS/DS-SILY and SIS groups (Figure 6E, 6F). 


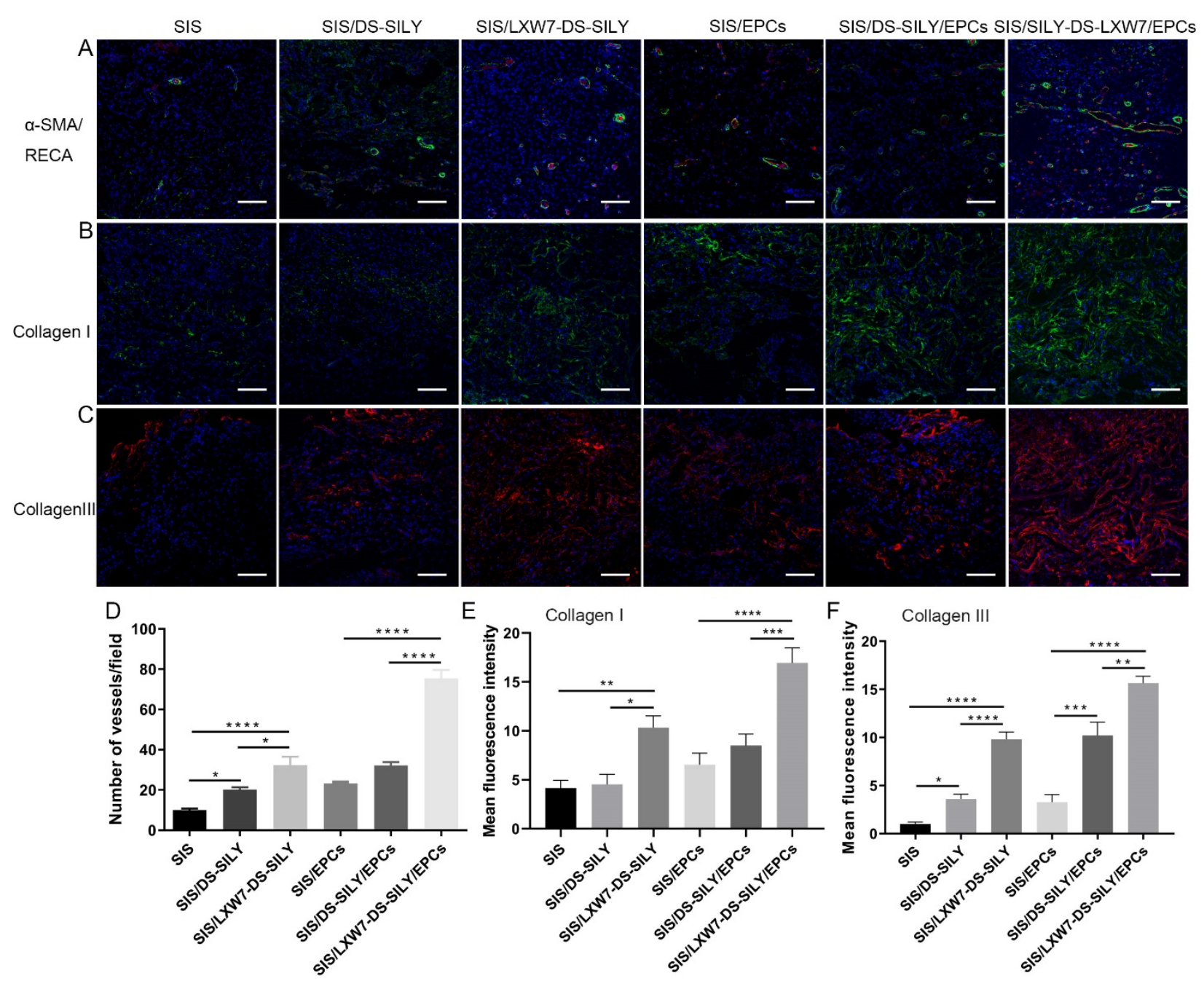

Figure 6 Neovascularization and collagen deposition evaluation of wounds treated by different scaffolds under non-ischemic condition.

A. Blood vessels stained with $\alpha$-SMA (green) and RECA (red) in wound bed at day 14 post surgery. B-C. Immunostaining of collagen I and collagen III expression in wound bed at day 14 post surgery. D-F. Quantification of the number of blood vessels/field (D), immunofluorescence intensity of collagen I (E) and collagen III (F). Data are expressed as mean \pm SEM. $* * p<0.01$, $* \mathrm{p}<0.05, \mathrm{n}=3$ per group.

3.4.4 LXW7-DS-SILY modified SIS scaffolds accelerated diabetic ischemic wound healing and supported ZDF-EPC survival 
To detect the effect of the different scaffolds on wound healing under ischemic conditions, we treated the ischemic wounds with SIS/LXW7-DS-SILY/EPCs, SIS/DS-SILY/EPCs, SIS/EPCs, SIS/LXW7-DS-SILY, SIS/DS-SILY or SIS. Digital photographs were obtained 0, 3, 7, 11, and 14 days after the operation (Figure 7A). The wound area in all groups decreased over time and the average observed wound closure rate was significantly higher in the SIS/LXW7-

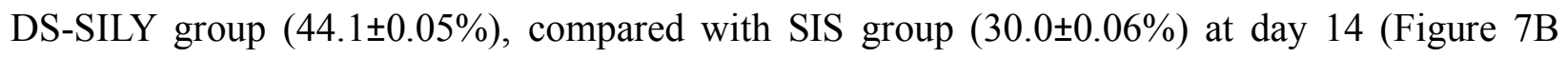
panel a). There was no difference between the SIS/DS-SILY (46.3 $\pm 0.08 \%)$ and the SIS/LXW7DS-SILY group $(44.1 \pm 0.05 \%)$ or SIS group $(30.0 \pm 0.06 \%)$. With respect to all groups loaded with ZDF-EPCs, the wounds treated with SIS/LXW7-DS-SILY/EPCs showed a wound closure rate of $61.6 \pm 0.05 \%$, significantly higher than that of the SIS/DS-SILY/EPCs $(43.5 \pm 0.05 \%)$ and SIS/EPCs $(34.8 \pm 0.05 \%)$ groups. There was no difference between SIS/DS-SILY/EPCs $(43.5 \pm 0.05 \%)$ and SIS/EPCs groups $(34.8 \pm 0.05 \%)$ (Figure 7B panel b). Finally, the SIS/LXW7DS-SILY/EPCs group showed a significantly higher healing rate than the SIS/LXW7-DS-SILY group highlighting the importance of the ZDF-EPCs in ischemic wound healing (Figure 7B panel c).

To investigate cell survival of transplanted cells in ischemic wounds, Td-Tomato/luciferinlabeled ZDF-EPCs were seeded on different scaffolds and imaged by IVIS (Figure 7C). The bioluminescence signal in SIS/LXW7-DS-SILY/EPCs group only showed a significantly higher intensity when compared to SIS/EPCs group at day 1 . The intensity in all three groups decreased to baseline by day 11 (Figure 7D). 

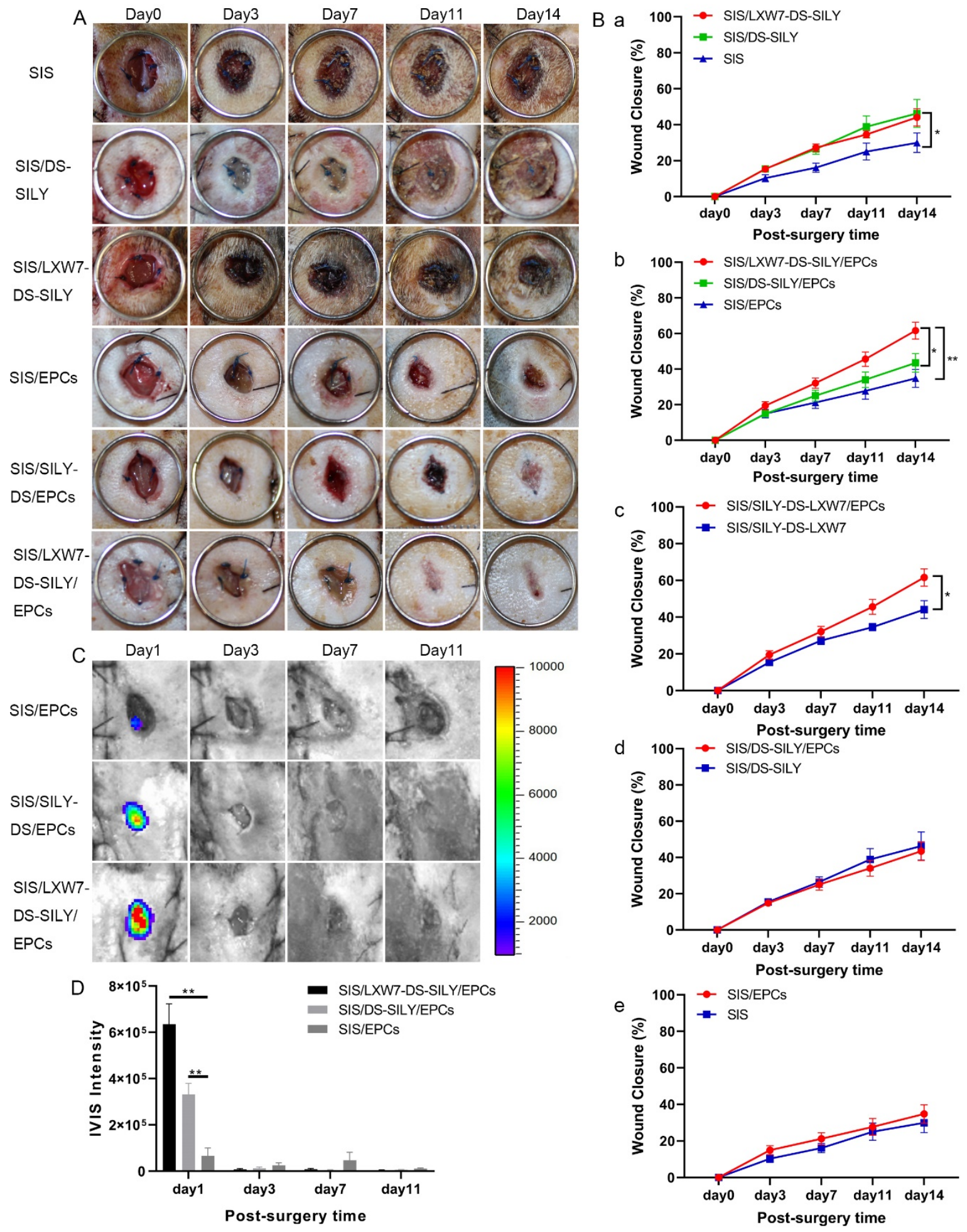

Figure 7 SIS/LXW7-DS-SILY constructs accelerate wound closure and support survival of ZDF-EPCs under ischemic condition. 
A. Representative images of healing in wounds treated with different groups at day $0,3,7,11$ and 14. B. Quantification of the wound closure rates of different groups. Data are expressed as mean \pm SEM. SIS, SIS/LXW7-DS-SILY, SIS/EPCs group: n=8; SIS/DS-SILY: n=7; SIS/DSSILY/EPCs: n=11; SIS/LXW7-DS-SILY/EPCs: n=12. C. Bioluminescence image of wounds transplanted with different modified scaffolds loaded with ZDF-EPCs. D. Quantification of the bioluminescence signals intensity (Most of the SIS scaffolds spontaneously fall off the wounds by day 11 ). Data are expressed as mean \pm SEM. $n=5$ per group. $* * p<0.01,{ }^{*} p<0.05$.

3.4.5 LXW7-DS-SILY modified SIS scaffolds accelerated wound healing and re-epithelialization in ischemic wound areas.

Overall, the wound healing quality of ischemic wounds is much worse than in non-ischemic wounds (Figure 8A). The SIS/LXW7-DS-SILY/EPCs group showed a trend toward more rapid re-epithelialization as compared to the SIS/EPCs group, as determined by shorter residual wound length (Figure 8C, 8D). However, no significant differences were found between the SIS/LXW7DS-SILY/EPCs and SIS/DS-SILY/EPCs groups. Without EPCs, SIS/LXW7-DS-SILY scaffolds alone still have a positive effect on re-epithelialization and wound length when compared to the SIS group. 

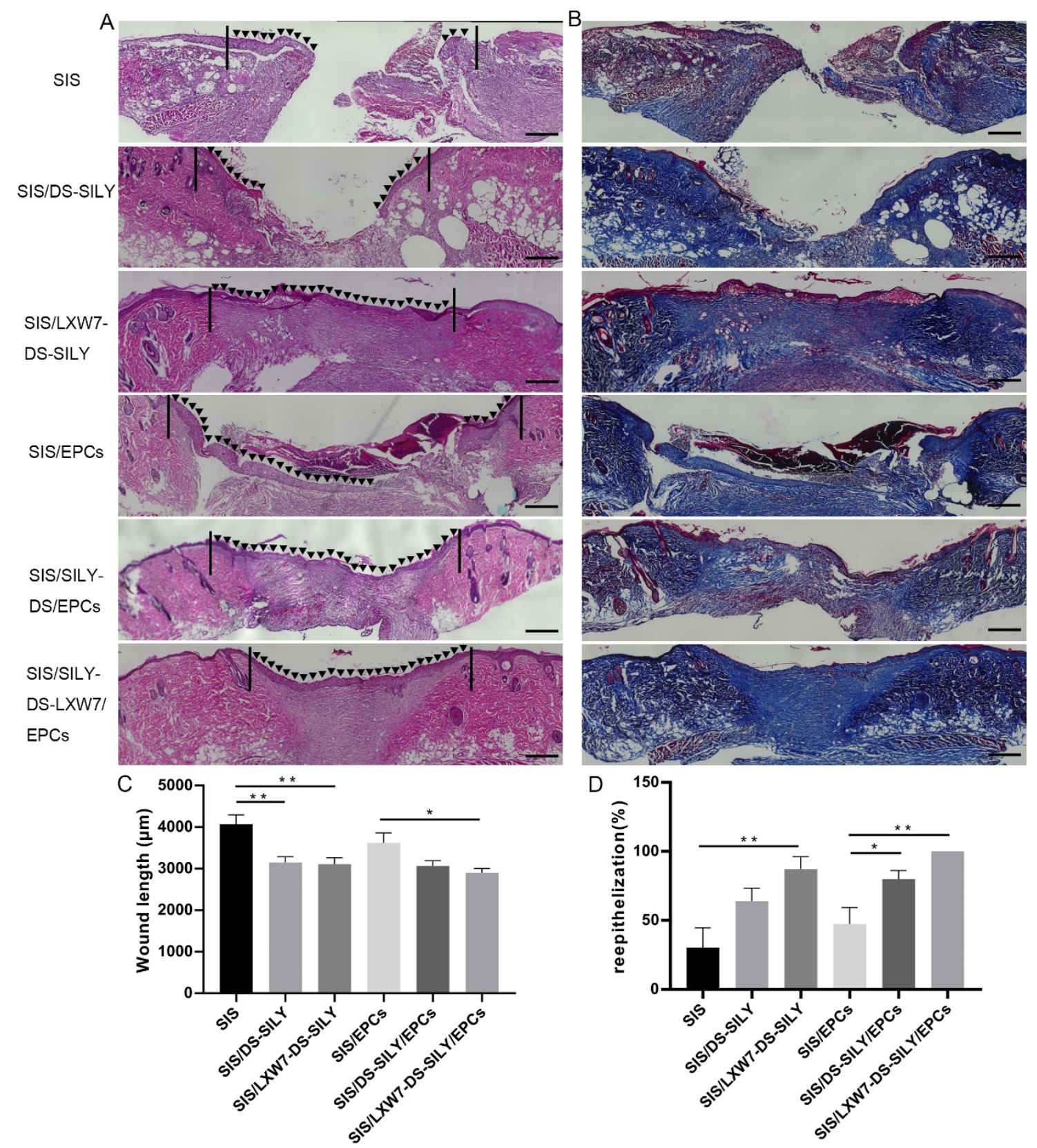

Figure 8 A. Representative images of H\&E staining of wounds at day 14 under ischemic condition. B. Representative images of Masson Trichrome staining of wounds at day 14 C-D. Quantification of the wound length (C) and re-epithelization rates (D) of different groups. Scale bar $=500 \mu \mathrm{m}$. Data are expressed as mean \pm SEM. $* * \mathrm{p}<0.01,{ }^{*} \mathrm{p}<0.05, \mathrm{n}=5$ per group.

3.4.6 LXW7-DS-SILY modified SIS scaffolds promote angiogenesis and stimulate collagen deposition in ischemic wounds 
To evaluate ischemic wound angiogenesis, we stained tissue sections for RECA-1 and $\alpha$ SMA to assess capillary densities at day 14 (Figure 9A). Wounds treated with SIS/LXW7-DSSILY scaffolds showed a significantly higher vessel number than SIS/DS-SILY and SIS scaffolds in the regenerated tissue (Figure 9D). When wounds were treated with different scaffolds seeded with ZDF-EPCs, SIS/LXW7-DS-SILY/EPCs promoted angiogenesis more than the other two groups (Figure 9D).

The result showed that irregular and fewer collagen fibers were formed during this time point for the SIS group as compared to other groups (Figure 8B). Enhanced collagen I and collagen III staining intensity was observed in SIS/LXW7-DS-SILY/EPCs treated wounds on day 14 when compared to the other wounds (Figure 9B, 9C). SIS/LXW7-DS-SILY showed a significantly higher collagen III intensity than the SIS/DS-SILY and SIS groups, and a higher collagen I intensity than the SIS group (Figure 9E, 9F).

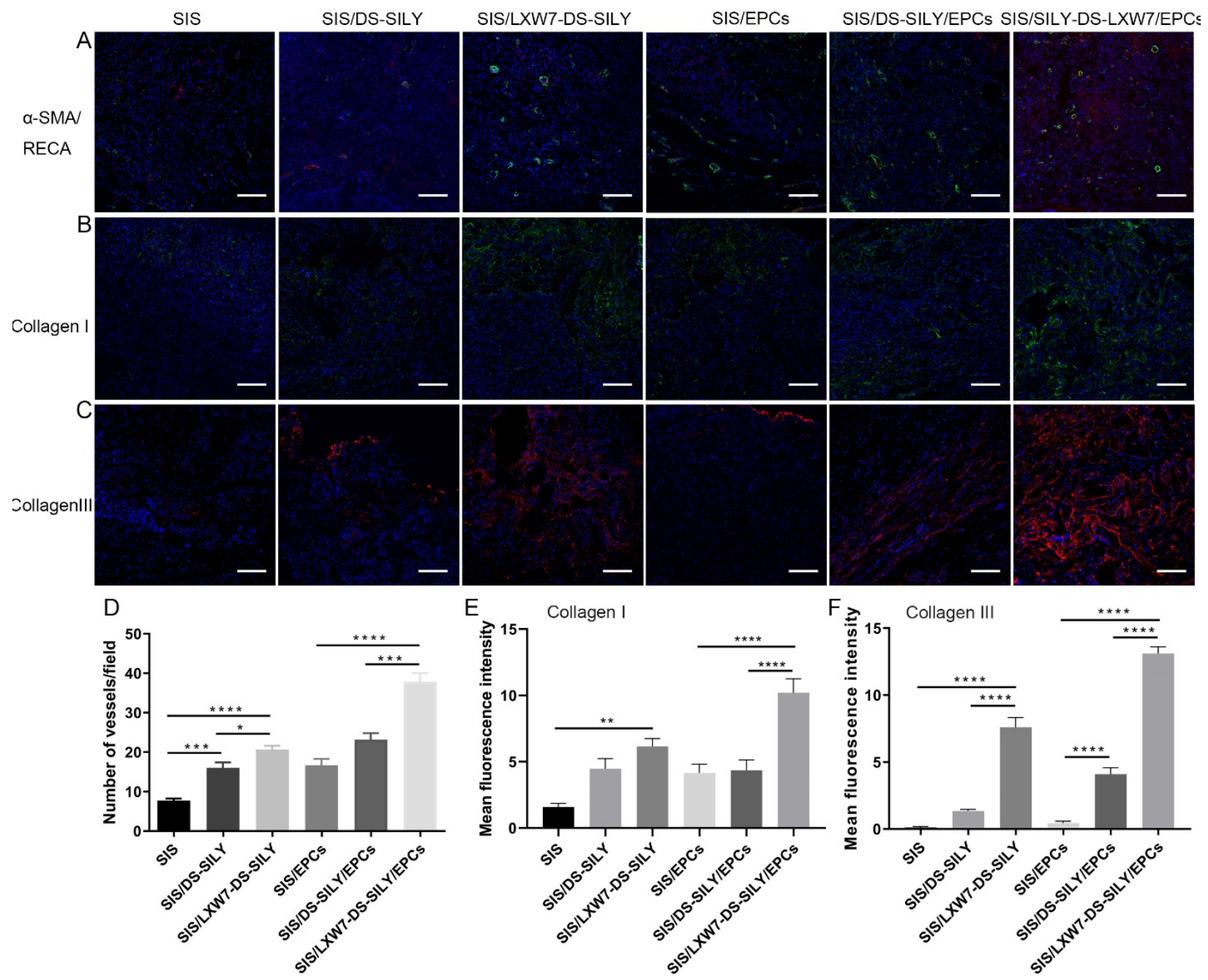


Figure 9 Neovascularization and collagen deposition evaluation of wounds treated by different scaffolds under ischemic condition.

A. Blood vessels stained with $\alpha$-SMA (green) and RECA (red) in wound bed at day 14 post surgery. B-C. Immunostaining of collagen I and collagen III expression in wound bed at day 14 post surgery. D-F. Quantification of the blood vessel number/field (D) and immunofluorescence intensity of collagen I (E) and collagen III (F). Data are expressed as mean \pm SEM. $* * * * \mathrm{p}<0.0001, * * * \mathrm{p}<0.001, * \mathrm{p}<0.05, \mathrm{n}=3$ per group.

\section{Discussion}

It is well established that impaired healing of diabetic wounds is affected by several factors, such as hypoxia, dysfunction of fibroblasts and epidermal cells, impaired angiogenesis and neovascularization, and high levels of metalloproteases [25]. Hyperinsulinemia in diabetic patients impairs the proliferation and tube formation of EPCs [26]. Stem cells and regenerative materials exhibit potentially therapeutic effects towards wound and tissue regeneration. EPCs play an especially important role in the wound healing process by promoting angiogenesis and facilitating wound closure [5]. Hence, finding an effective way to augment the function of EPCs isolated from diabetic patients and delivering them to the wound site will be critical for accelerating the healing of diabetic wounds via autologous therapy.

The streptozotocin (STZ)-induced diabetic rodent model with non-ketosis hyperglycemia is the most widely used model for diabetic wound healing research [27]. However, the STZ model represents type 1 diabetes and has limitations such as organ toxicity, low stability, and impaired weight gain in animals [28]. Since type 2 diabetes account for the majority of patients with diabetes, and given the prevalence of ischemic wounds in type 2 diabetic patients, animal models that better recapitulate the pathophysiological situations of type 2 diabetic ischemic wounds serve as a better option for evaluating the function of innovative treatments for diabetic ischemic wound healing. In this study, we designed and developed a regenerative bioscaffold (SIS/LXW7DS-SILY) loaded with EPCs and tested the therapeutic efficacy by localized topical application of the scaffold at excisional wound sites that were created to mimic the highly ischemic condition of the clinical DFU. We chose to use the Zucker diabetic fat (ZDF) rat model to evaluate the therapeutic effects of the regenerative treatment for diabetic ischemic wounds. The ZDF rat is a well-characterized model of the metabolic syndrome and the pathophysiologic 
mechanism of this model is attributed to the missense mutation of leptin receptor [28, 29]. ZDF rats exhibit obesity with diabetes and are characterized by hyperglycemia, insulin resistance, hyperlipidemia and impaired glucose tolerance [28], accompanied with delayed wound healing, impeded blood flow, and diabetic nephropathy [30-32]. ZDF rats become obese at around 4 weeks of age and develop type 2 diabetes at 8-12 weeks of age [28]. Additionally, in order to mimic the highly ischemic condition of the clinical DFU in this diabetic rat model, we inserted a silicone sheet beneath the pedicle flap containing the excisional wound to ensure no reperfusion from underlying tissue [23].

The wound healing mechanisms between rodents and humans are different due to anatomical variations. In rodents, healing occurs by wound contraction because of the paniculosus carnosus layer that is situated directly below the skin. In contrast, wound healing in humans occurs via re-epithelization and granulation tissue formation [34]. However, Slavkovsky, et al. [31] showed that the contraction of diabetic wounds in ZDF rats is impaired, and wound healing predominantly occurs by re-epithelialization at the wound site. To mimic this in our study, scaffolds were immobilized to the wounds with silk threads which can reduce wound contraction or enlargement. Together, the ZDF rat model developed in this study is an effective model to study the therapeutic efficacy of functionalized scaffolds for human diabetic ischemic wound healing.

Using this model, we showed that under the non-ischemic condition, the SIS/LXW7-DSSILY/EPCs group displayed a significantly faster rate of wound closure and shorter wound lengths but showed no difference in the re-epithelialization process compared to SIS/DSSILY/EPCs and the SIS/LXW7-DS-SILY groups. Under the ischemic condition, SIS/LXW7-DSSILY/EPCs group again displayed a significantly faster rate of wound closure and shorter wound lengths, and in addition a rapid re-epithelialization compared to other groups. These results together suggest that LXW7 and EPCs improve the wound healing process. From our previous studies, we found that the LXW7-modified small diameter vascular grafts significantly promoted EPC/EC recruitment and rapidly achieved endothelialization [24]. Our results obtained here suggest that LXW7 may aid in the recruitment of endogenous EPCs in the non-ischemic condition and increases the EPC survival in the functionalized scaffold. It is also possible that the EPCs secrete paracrine factors that stimulate proximal ECs, and thus promote angiogenesis and improve wound healing in both conditions [35]. Interestingly, the healing of the SIS/LXW7-DS- 
SILY/EPCs group is significantly better than the SIS/LXW7-DS-SILY group under the ischemic condition but shows no difference under the non-ischemic condition. SIS/LXW7-DS-SILY alone facilitated improved healing effect under the non-ischemic condition. Because we know that LXW7 can effectively recruit endogenous EPCs [24], it is reasonable to speculate that LXW7 can promote wound healing by recruiting endogenous EPCs to wound sites under sufficient blood supply. In highly ischemic diabetic wound sites, there is insufficient blood supply; therefore, endogenous EPCs cannot be recruited as effectively to the wound. Transplanted EPCs may also serve to help in ischemic wound healing via releasing factors that support angiogenesis of the existing blood vessels. Even though the EPCs in the SIS/LXW7-DS-SILY/EPCs group were present in high numbers up to 24 hours and decreased thereafter, the increase in healing rate suggest that their presence for even a short period of time is sufficient to initiate tissue granulation, leading to re-epithelialization. The bioluminescence intensity of all the groups decreased to baseline at day 11. This is due in part to most of the SIS scaffolds spontaneously falling off at day 11 .

It is well known that wound neovascularization is essential in order to generate sufficient blood supply to the wound area for constant supply of oxygen and nutrition, the two important factors that promote healing [27]. The results obtained from the ischemic and non-ischemic wounds treated with the SIS/LXW7-DS-SILY/EPCs scaffolds showed an enhancement in angiogenesis when compared with other groups. Our previous study indicated that LXW7 can bind to EPCs and ECs via $\alpha v \beta 3$ integrin and enhance EC biological functions by increasing phosphorylation of VEGF receptor 2 (VEGF-R2) and activating mitogen-activated protein kinase (MAPK) ERK1/2 [18]. Our in vitro data also showed that LXW7 and LXW7-DS-SILY modified surfaces promoted attachment and growth of ZDF-EPCs (Figure 3). The results that SIS/LXW7DS-SILY scaffolds alone had a positive effect on angiogenesis suggest that the LXW7 ligand potentially recruited and interacted with endogenous EPCs/ECs, and thus successfully promoted angiogenesis, neovascularization and wound healing in the diabetic wounds.

Proper collagen deposition and remodeling improve skin elasticity and toughness and greatly influence the outcomes of wound healing [36]. From immunohistological analyses (Figure 9), we observed that all the modified scaffolds displayed abundant and relatively wellorganized collagen fibers. Since in our previous studies, DS-SILY showed the ability to improve collagen organization [14], we expect that the modification with functional ligands DS-SILY or 
LXW7-DS-SILY may exert positive effects on the ECM molecules in the wound areas, such as limiting collagen degradation and maintaining the architecture of collagen fibers.

Collagen I and III are central components of the dermal ECM with collagen I constituting $80-85 \%$, and collagen III constituting $8-11 \%$ of the dermal ECM, and both playing a vital role in wound healing [37]. Previous studies revealed that sufficient amounts of collagen III in the early wound healing process would accelerate healing and result in scarless skin. [38]. It is known that ischemic wounds induce excessive production and over-activity of MMPs in the chronic wound environment, causing rapid degradation of new granulation tissue and countering the healing process [38-40]. DS-SILY was previously shown to improve collagen organization via inhibition of MMP-mediated collagen degradation [14]. In this study, the DS-SILY and LXW7-DS-SILY ligand modified groups displayed a significantly higher intensity of collagen I or III deposition when compared to the control group, suggesting that the ligand may protect the matrix from MMP degradation, and thus suppress scar formation and mimic the wound healing process as in normal skin. Future studies will be needed to further assess this aspect of the wound healing induced with LXW7-DS-SILY

In summary, this study demonstrates a novel therapeutic approach to accelerate diabetic ischemic wound healing by application of a functionalized combinatorial scaffold consisting of three functional components: 1) LXW7-DS-SILY, a bifunctional ligand; 2) SIS-ECM scaffold; and 3) EPCs. Further studies are necessary to explore matrix deposition at different time points, to investigate the cell populations recruited by the functionalized scaffolds, and to elucidate the potential mechanisms of LXW7 supporting the survival of transplanted EPCs, EPC paracrine signaling, and explore the potential application of this therapeutic approach to other diseases and conditions. Experiments involving large animals such as pigs which are more similar to human's skin structure and function to further validate the safety and efficacy of this therapeutic approach will be warranted before any clinical use.

\section{Conclusions}

This study has successfully demonstrated the importance of LXW7-DS-SILY functionalized SIS scaffolds in enhancing wound healing in a type 2 diabetic rat ischemic skin flap model. EPCs cultured on SIS/LXW7-DS-SILY scaffolds showed enhanced attachment and growth. In addition, the data indicated that wound closure, re-epithelialization, angiogenesis and collagen deposition were enhanced in the diabetic ischemic wounds treated with the SIS-ECM scaffold 
functionalized with LXW7-DS-SILY and EPCs. Thus, LXW7-DS-SILY combined with an ECM scaffold and EPCs could be a promising novel treatment to accelerate healing of diabetic ischemic wounds, thereby reducing limb amputation and mortality rates of diabetic patients.

\section{Acknowledgements}

We thank Cook Biotech Inc. for their generous gifting of SIS-ECM material. This work was partially supported by California Institute for Regenerative Medicine grant number DISC110516-0, and Shriners Hospitals for Children developmental research award grant number 87200-NCA-19. This work utilized the Combinatorial Chemistry Shared Resource at the UC Davis Comprehensive Cancer Center which is supported by NCI P30CA093373 Cancer Center Support Grant.

\section{Data Availability Statement}

All data generated or analyzed during this study are included in this published article. 


\section{References}

[1] International Diabetes Federation, IDF Diabetes Atlas 9th edition. http://www.diabetesatlas.org, 2019.

[2] L.R. Kalan, J.S. Meisel, M.A. Loesche, J. Horwinski, I. Soaita, X. Chen, A. Uberoi, S.E. Gardner, E.A. Grice. Strain- and Species-Level Variation in the Microbiome of Diabetic Wounds Is Associated with Clinical Outcomes and Therapeutic Efficacy. Cell Host Microbe. 25 (2019) 641-655.e5. https://doi.org/10.1016/j.chom.2019.03.006.

[3] A. Hingorani, G.M. Lamuraglia, P. Henke, M.H. Meissner, L. Loretz, K.M. Zinszer, V.R. Driver, R. Frykberg, T.L. Carman, W. Marston, J.L. Mills, M.H. Murad. The management of diabetic foot: A clinical practice guideline by the Society for Vascular Surgery in collaboration with the American Podiatric Medical Association and the Society for Vascular Medicine. J. Vasc. Surg. 63 (2016) 3S-21S. https://doi.org/10.1016/j.jvs.2015.10.003.

[4] C. Wang, Q. Wang, W. Gao, Z. Zhang, Y. Lou, H. Jin, X. Chen, B. Lei, H. Xu, C. Mao. Highly Efficient Local Delivery of Endothelial Progenitor Cells Significantly Potentiates Angiogenesis and Full-thickness Wound Healing. Acta Biomater. 69 (2018) 156-169. https://doi.org/10.1016/j.actbio.2018.01.019.

[5] S. Kanji, H. Das. Advances of Stem Cell Therapeutics in Cutaneous Wound Healing and Regeneration. Mediators Inflamm. 2017 (2017). https://doi.org/10.1155/2017/5217967.

[6] A.C.D.O. Gonzalez, Z.D.A. Andrade, T.F. Costa, A.R.A.P. Medrado. Wound healing - A literature review. An. Bras. Dermatol. 91 (2016) 614-620. https://doi.org/10.1590/abd1806-4841.20164741.

[7] K. Kaushik, A. Das. Endothelial progenitor cell therapy for chronic wound tissue regeneration. Cytotherapy. 21 (2019) 1137-1150.

https://doi.org/10.1016/j.jcyt.2019.09.002.

[8] O.C. Velazquez. Angiogenesis and vasculogenesis: Inducing the growth of new blood vessels and wound healing by stimulation of bone marrow-derived progenitor cell mobilization and homing. J. Vasc. Surg. 45 (2007) A39-A47. https://doi.org/10.1016/j.jvs.2007.02.068.

[9] Z.J. Liu, O.C. Velazquez. Hyperoxia, endothelial progenitor cell mobilization, and diabetic wound healing. Antioxidants Redox Signal. 10 (2008) 1869-1882. https://doi.org/10.1089/ars.2008.2121.

[10] L. Wang, W. Wang, J. Liao, F. Wang, J. Jiang, C. Cao, S. Li. Novel bilayer wound dressing composed of SIS membrane with SIS cryogel enhanced wound healing process. Mater. Sci. Eng. C. 85 (2018) 162-169. https://doi.org/10.1016/j.msec.2017.11.024. 
[11] L. Lankford, Y.J. Chen, Z. Saenz, P. Kumar, C. Long, D. Farmer, A. Wang. Manufacture and preparation of human placenta-derived mesenchymal stromal cells for local tissue delivery. Cytotherapy. 19 (2017) 680-688. https://doi.org/10.1016/j.jcyt.2017.03.003.

[12] G.L.B. P.S. Wolfe, S.A. Sell. Natural and synthetic scaffolds. Tissue Eng. (2011) 41-47.

[13] P. Zhao, X. Li, Q. Fang, F. Wang, Q. Ao, X. Wang, X. Tian, H. Tong, S. Bai, J. Fan. Surface modification of small intestine submucosa in tissue engineering. Regen. Biomater. 7 (2020) 339-348. https://doi.org/10.1093/rb/rbaa014.

[14] K. Stuart, J. Paderi, P.W. Snyder, L. Freeman, A. Panitch. Collagen-Binding Peptidoglycans Inhibit MMP Mediated Collagen Degradation and Reduce Dermal Scarring. PLoS One. 6 (2011). https://doi.org/10.1371/journal.pone.0022139.

[15] C.C. Reed, R. V. Iozzo. The role of decorin in collagen fibrillogenesis and skin homeostasis. Glycoconj. J. 19 (2002) 249-255. https://doi.org/10.1023/A:1025383913444.

[16] K.G. Vogel, J.A. Trotter. The Effect of Proteoglycans on the Morphology of Collagen Fibrils Formed In Vitro. Top. Catal. 7 (1987) 105-114. https://doi.org/10.1016/S0174$173 \mathrm{X}(87) 80002-\mathrm{X}$.

[17] P.G. Scott, C.M. Dodd, E.E. Tredget. A. Ghahary, F. Rahemtulla, Chemical characterization and quantification of proteoglycans in human post-burn hypertrophic and mature scars. Clin. Sci. 90 (1996) 417-425. https://doi.org/10.1042/cs0900417.

[18] D. Hao, W. Xiao, R. Liu, P. Kumar, Y. Li, P. Zhou, F. Guo, D.L. Farmer, K.S. Lam, F. Wang, A. Wang. Discovery and Characterization of a Potent and Specific Peptide Ligand Targeting Endothelial Progenitor Cells and Endothelial Cells for Tissue Regeneration. ACS Chem. Biol. 12 (2017) 1075-1086. https://doi.org/10.1021/acschembio.7b00118.

[19] M.J. Webber, J. Tongers, C.J. Newcomb, K.T. Marquardt, J. Bauersachs, D.W. Losordo, S.I. Stupp. Supramolecular nanostructures that mimic VEGF as a strategy for ischemic tissue repair.Proc Natl Acad Sci U S A. 2011 Aug 16;108(33):13438-43. doi: 10.1073/pnas.1016546108.

[20] J.E. Paderi, A. Panitch. Design of a synthetic collagen-binding peptidoglycan that modulates collagen fibrillogenesis. Biomacromolecules. 9 (2008) 2562-2566. https://doi.org/10.1021/bm8006852.

[21] W. Xiao, Y. Wang, E.Y. Lau, J. Luo, N. Yao, C. Shi, L. Meza, H. Tseng, Y. Maeda, P. Kumaresan, R. Liu, F.C. Lightstone, Y. Takada, K.S. Lam. The use of one-bead onecompound combinatorial library technology to discover high-affinity $\alpha v \beta 3$ integrin and cancer targeting arginine-glycine-aspartic acid ligands with a built-in handle. Mol. Cancer Ther. 9 (2010) 2714-2723. https://doi.org/10.1158/1535-7163.MCT-10-0308. 
[22] Tanaya Walimbe Siqi He Aijun Wang Alyssa Panitch. Collagen Binding Proteoglycan Mimetics to Promote Ischemic Wound Healing. in: BMES Annu. Conf., 2019.

[23] A.N. Trujillo, S.L. Kesl, J. Sherwood, M. Wu, L.J. Gould. Demonstration of the rat ischemic skin wound model. J. Vis. Exp. 2015 (2015) 1-7. https://doi.org/10.3791/52637.

[24] D. Hao, Y. Fan, W. Xiao, R. Liu, C. Pivetti, T. Walimbe, F. Guo, X. Zhang, D.L. Farmer, F. Wang, A. Panitch, K.S. Lam, A. Wang. Rapid endothelialization of small diameter vascular grafts by a bioactive integrin-binding ligand specifically targeting endothelial progenitor cells and endothelial cells. Acta Biomater. 108 (2020) 178-193. https://doi.org/10.1016/j.actbio.2020.03.005.

[25] S. Guo, L.A. DiPietro. Critical review in oral biology \& medicine: Factors affecting wound healing. J. Dent. Res. 89 (2010) 219-229.

https://doi.org/10.1177/0022034509359125.

[26] Q. Tan, Y. Li, X. Li, S. Zhang. Hyperinsulinemia impairs functions of circulating endothelial progenitor cells. Acta Diabetol. 56 (2019) 785-795.

https://doi.org/10.1007/s00592-019-01314-9.

[27] Y. Kato, T. Iwata, S. Morikawa, M. Yamato, T. Okano, Y. Uchigata. Allogeneic transplantation of an adipose-derived stem cell sheet combined with artificial skin accelerates wound healing in a rat wound model of type 2 diabetes and obesity. Diabetes. 64 (2015) 2723-2734. https://doi.org/10.2337/db14-1133.

[28] N. Goonoo, A. Bhaw-Luximon. Analyzing polymeric nanofibrous scaffold performances in diabetic animal models for translational chronic wound healing research. Nanotechnol. Rev. 6 (2017) 583-600. https://doi.org/10.1515/ntrev-2017-0162.

[29] P.N. Chander, O. Gealekman, S. V. Brodsky, S. Elitok, A. Tojo, M. Crabtree, S.S. Gross, M.S. Goligorsky. Nephropathy in Zucker diabetic fat rat is associated with oxidative and nitrosative stress: Prevention by chronic therapy with a peroxynitrite scavenger ebselen. J. Am. Soc. Nephrol. 15 (2004) 2391-2403. https://doi.org/10.1097/01.ASN.0000135971.88164.2C.

[30] M.S. Phillips, Q. Liu, H.A. Hammond, V. Dugan, P.J. Hey, C.T. Caskey, J.F. Hess. Leptin receptor missense mutation in the fatty zucker rat. Nat. Genet. 13 (1996) 18-19. https://doi.org/10.1038/ng0596-18.

[31] R. Slavkovsky, R. Kohlerova, V. Tkacova, A. Jiroutova, B. Tahmazoglu, V. Velebny, M. Rezačová, L. Sobotka, J. Kanta. Zucker diabetic fatty rat: A new model of impaired cutaneous wound repair with type II diabetes mellitus and obesity. Wound Repair Regen. 19 (2011) 515-525. https://doi.org/10.1111/j.1524-475X.2011.00703.x. 
[32] L.J. Coppey, J.S. Gellett, E.P. Davidson, J.A. Dunlap, M.A. Yorek. Changes in endoneurial blood flow, motor nerve conduction velocity and vascular relaxation of epineurial arterioles of the sciatic nerve in ZDF-obese diabetic rats. Diabetes. Metab. Res. Rev. 18 (2002) 49-56. https://doi.org/10.1002/dmrr.257.

[33] C.L. Oltman, L.J. Coppey, J.S. Gellett, E.P. Davidson, D.D. Lund, M.A. Yorek. Progression of vascular and neural dysfunction in sciatic nerves of Zucker diabetic fatty and Zucker rats. Am. J. Physiol. - Endocrinol. Metab. 289 (2005) 113-123. https://doi.org/10.1152/ajpendo.00594.2004.

[34] P.B. Milan, N. Lotfibakhshaiesh, M.T. Joghataie, J. Ai, A. Pazouki, D.L. Kaplan, S. Kargozar, N. Amini, M.R. Hamblin, M. Mozafari, A. Samadikuchaksaraei. Accelerated wound healing in a diabetic rat model using decellularized dermal matrix and human umbilical cord perivascular cells. Acta Biomater. 45 (2016) 234-246. https://doi.org/10.1016/j.actbio.2016.08.053.

[35] S. Xu, J. Zhu, L. Yu, G. Fu. Endothelial Progenitor Cells : Current Development of Their Paracrine Factors in Cardiovascular Therapy. J Cardiovasc Pharmacol. 59 (2012) 387-396. doi: 10.1097/FJC.0b013e3182440338.

[36] X. Zhao, H. Wu, B. Guo, R. Dong, Y. Qiu, P.X. Ma. Biomaterials Antibacterial antioxidant electroactive injectable hydrogel as self- healing wound dressing with hemostasis and adhesiveness for cutaneous wound healing. Biomaterials. 122 (2017) 3447. https://doi.org/10.1016/j.biomaterials.2017.01.011.

[37] E. Davison-kotler, W.S. Marshall, E. Garc. Sources of Collagen for Biomaterials in Skin Wound Healing. Bioengineering (Basel). (2019) 1-15. doi: 10.3390/bioengineering6030056.

[38] C. Wang, M. Wang, T. Xu, X. Zhang, C. Lin, W. Gao, H. Xu, B. Lei, C. Mao. Engineering bioactive self-healing antibacterial exosomes hydrogel for promoting chronic diabetic wound healing and complete skin regeneration. Theranostics. 9 (2019) 65-76. https://doi.org/10.7150/thno.29766.

[39] P. Yang, Q. Pei, T. Yu, Q. Chang, D. Wang, M. Gao, X. Zhang, Y. Liu. Compromised wound healing in ischemic type 2 diabetic rats. PLoS One. 11 (2016) 1-19. https://doi.org/10.1371/journal.pone.0152068.

[40] S. Eming, H. Smola, B. Hartmann, G. Malchau, R. Wegner, T. Krieg, S. Smola-hess. The inhibition of matrix metalloproteinase activity in chronic wounds by a polyacrylate superabsorber. Biomaterials. 29 (2008) 2932-2940. doi:

10.1016/j.biomaterials.2008.03.029. 
[41] N. Krishnan, B. Velramar, G.C. Abraham, N. Duraisamy, R. Pandiyan, RK. Velu. Effect of biogenic silver nanocubes on matrix metalloproteinases 2 and 9 expressions in hyperglycemic skin injury and its impact in early wound healing in streptozotocin-induced diabetic mice, Mater Sci Eng C Mater Biol Appl. 2018 Oct 1;91:146-152. doi: 10.1016/j.msec.2018.05.020. 